\title{
Combined Cytological and Transcriptomic Analysis Reveals a Nitric Oxide Signaling Pathway Involved in Cold-Inhibited Camellia sinensis Pollen Tube Growth
}

\author{
Weidong Wang ${ }^{1}$, Xianyong Sheng ${ }^{2}$, Zaifa Shu ${ }^{1}$, Dongqin Li $^{1}$, Junting Pan ${ }^{1}$, Xiaoli Ye ${ }^{1}$, \\ Pinpin Chang ${ }^{1}$, Xinghui $L{ }^{1}{ }^{1}$ and Yuhua Wang ${ }^{1 *}$ \\ ${ }^{1}$ College of Horticulture, Nanjing Agricultural University, Nanjing, China, ${ }^{2}$ College of Life Sciences, Capital Normal University, \\ Beijing, China
}

OPEN ACCESS

Edited by:

Keqiang Wu,

National Taiwan University, Taiwan

Reviewed by:

Gabriela Carolina Pagnussat, Universidad Nacional de Mar del

Plata, Argentina

Xinchao Wang,

Chinese Academy of Agricultural

Sciences, China

Xiao Chun Wan,

Anhui Agriculture University, China

*Correspondence:

Yuhua Wang

wangyuhua@njau.edu.cn

Specialty section:

This article was submitted to Plant Genetics and Genomics,

a section of the journal

Frontiers in Plant Science

Received: 28 January 2016

Accepted: 24 March 2016

Published: 14 April 2016

Citation:

Wang W, Sheng X, Shu Z, Li D, Pan J,

$Y \in X$, Chang $P, L i X$ and Wang $Y$

(2016) Combined Cytological and Transcriptomic Analysis Reveals a

Nitric Oxide Signaling Pathway

Involved in Cold-Inhibited Camellia sinensis Pollen Tube Growth

Front. Plant Sci. 7:456.

doi: 10.3389/fpls.2016.00456
Nitric oxide (NO) as a signaling molecule plays crucial roles in many abiotic stresses in plant development processes, including pollen tube growth. Here, the signaling networks dominated by NO during cold stress that inhibited Camellia sinensis pollen tube growth are investigated in vitro. Cytological analysis show that cold-induced NO is involved in the inhibition of pollen tube growth along with disruption of the cytoplasmic $\mathrm{Ca}^{2+}$ gradient, increase in ROS content, acidification of cytoplasmic $\mathrm{pH}$ and abnormalities in organelle ultrastructure and cell wall component distribution in the pollen tube tip. Furthermore, differentially expressed genes (DEGs)-related to signaling pathway, such as NO synthesis, cGMP, $\mathrm{Ca}^{2+}, \mathrm{ROS}, \mathrm{pH}$, actin, cell wall, and MAPK cascade signal pathways, are identified and quantified using transcriptomic analyses and qRT-PCR, which indicate a potential molecular mechanism for the above cytological results. Taken together, these findings suggest that a complex signaling network dominated by NO, including $\mathrm{Ca}^{2+}$, ROS, pH, RACs signaling and the crosstalk among them, is stimulated in the $C$. sinensis pollen tube in response to cold stress, which further causes secondary and tertiary alterations, such as ultrastructural abnormalities in organelles and cell wall construction, ultimately resulting in perturbed pollen tube extension.

Keywords: Camellia sinensis, pollen tube growth, nitric oxide, cold stress, signaling pathway

\section{INTRODUCTION}

Low temperature is a major factor that significantly constrains the life cycle of higher plants, including germination, growth, development, flowering, and seed setting (Klemens et al., 2014; Maruyama et al., 2014). Among these processes, reproductive processes, particularly pollen tube growth, are negatively regulated by low temperatures (Hedhly, 2011). According to previous reports, cold stress significantly reduces the pollen tube growth of Cicer arietinum (Srinivasan et al., 1999) and Pyrus bretschneideri (Gao et al., 2014) and disrupts the morphology of the pollen tube tip zone (Srinivasan et al., 1999). Recently, the actin cytoskeleton, endocytosis and some signaling molecules, such as the calcium ion $\left(\mathrm{Ca}^{2+}\right)$ and reactive oxygen species (ROS), have been implicated in the cold stress-inhibited pollen tube growth in vitro (Wu et al., 2012; Gao et al., 2014). However, 
the underlying basis of the cellular mechanisms of pollen tube growth under cold stress remains largely unknown.

Nitric oxide (NO) is a highly active gaseous signaling molecule that plays crucial roles in many key physiological processes in plants, including seed germination, photo morphogenesis, mitochondrial activity, leaf expansion, root growth, regulation of stomatal movement, fruit maturation, senescence and iron metabolism, etc. (Lanteri et al., 2008; Neill et al., 2008; Sanz et al., 2015). Furthermore, many investigations have revealed that NO plays an important role in the reproductive processes of higher plants, including flower bud differentiation, flowering induction, fertilization and seed setting, particularly pollen tube tip growth (Prado et al., 2008; Domingos et al., 2015). For example, NO participated in self-incompatibility-induced programmed cell death (PCD) in the Papaver rhoeas pollen tube through interactions with $\mathrm{Ca}^{2+}$, ROS and actin signaling (Wilkins et al., 2011), and there was a suspected potential association between $\mathrm{NO}$ and other signaling factors, such as the MAPK cascade and cytoplasmic $\mathrm{pH}$, in this process (Wilkins et al., 2014, 2015). Similarly, NO was found to modulate both the influx of extracellular $\mathrm{Ca}^{2+}$ and actin filament organization during cell wall construction to regulate the tip growth of Pinus bungeana pollen tubes (Wang et al., 2009). Moreover, NO is also involved in the tolerance of plants to various abiotic stresses (Qiao et al., 2014), such as high salt (Qiao et al., 2009), heat (Xuan et al., 2010), drought (Liao et al., 2012), heavy metals (Saxena and Shekhawat, 2013; Kováčik et al., 2014), and UV-B irradiation stress (Tossi et al., 2009), particularly in cold acclimation and freezing tolerance of plants. For example, Majláth et al. (2012) reported an increased production of NO in Triticum aestivum roots after exposure to cold stress, but a decrease of NO content was found in Capsicum annuum leaves when they were exposed to low temperatures (Airaki et al., 2012). Recently, NO has been proposed to protect plants from chilling injury by increasing their antioxidant defenses and thereby preventing ROS damage; NO stimulated the activity of S-nitrosylated proteins in Brassica juncea under cold stress (Sehrawat et al., 2013a). Additionally, NO participates in cold-triggered root growth inhibition by regulating the content of long-chain bases and the expression of cold-responsive genes (Guillas et al., 2013; Puyaubert and Baudouin, 2014). These data therefore suggest that there is a potential signal regulatory network that depends on $\mathrm{NO}$ in a plant's response to cold stress; further investigation is required to clarify the underlying mechanisms of this process.

Above all, NO is thought to act as a core signaling molecule in the cold stress-mediated inhibition of pollen tube growth, and this hypothesis has been supported by physiological and pharmacological findings in our previous research, which showed that NO production from NO synthase (NOS)-like activity decreased cold-responsive pollen germination, inhibited pollen tube growth and reduced proline (Pro) accumulation, partly via the cGMP signaling pathway in Camellia sinensis (Wang et al., 2012). However, the role of the NO-dependent complex signaling network, including cGMP, $\mathrm{Ca}^{2+}$, ROS, actin, and $\mathrm{pH}$ signaling and the cross-talk among them, in the process of cold stressinhibited pollen tube growth, has not yet been elucidated. In the present study, we also investigated the signal transduction roles of $\mathrm{NO}$ during pollen tube elongation in response to cold stress in C. sinensis. Specifically, we focused on cold-induced NO that is involved in inhibiting the tip growth of the pollen tube, in addition to several linked cellular features that are essential for the NO signaling pathway under cold tolerance, including the cytoplasmic $\mathrm{Ca}^{2+}$ gradient, the ROS concentrations, the acidification of the cytoplasm, the tip ultrastructure, and the composition of the cell wall. Moreover, we also performed the identification of differentially expressed genes in the coldinduced NO signaling pathway in C. sinensis pollen tubes, including genes involved in NO synthesis, cGMP, $\mathrm{Ca}^{2+}, \mathrm{ROS}, \mathrm{pH}$, actin, the cell wall, and the MAPK cascade, using transcriptomic analyses, which provided insight into the molecular mechanisms that underlie the above events. These data provide further insights into the regulation of NO signaling in the pollen tube response to cold stress in $C$. sinensis.

\section{MATERIALS AND METHODS}

\section{Plant Material and In vitro Pollen Culture}

Mature pollen was collected from " $C$. sinensis (L.) O. Kuntze cv. Longjingchangye" tea plants. The pollen was pre-incubated in standard culture medium [containing $30 \mathrm{mM} \mathrm{MES,} \mathrm{5 \%} \mathrm{(w/v)}$ sucrose, $0.01 \%(\mathrm{w} / \mathrm{v}) \mathrm{H}_{3} \mathrm{BO}_{3}, 0.05 \%$ (w/v) $\mathrm{Ca}\left(\mathrm{NO}_{3}\right)_{2} \cdot 4 \mathrm{H}_{2} \mathrm{O}$, and $5 \%\left(\mathrm{w} / \mathrm{v}\right.$ ) PEG 4000, $\mathrm{pH} 6.0$ ] at $25^{\circ} \mathrm{C}$ in the dark for $30 \mathrm{~min}$ in vitro. For cold stress treatment, the pre-incubated pollen was transferred and maintained at $4^{\circ} \mathrm{C}$ in the dark. In addition, NO donor DEA NONOate $(25 \mu \mathrm{M})$ and NO scavenger 2-(4carboxyphenyl)-4,4,5,5-tetramethylimidazoline-1-oxyl-3-oxide (cPTIO, $200 \mu \mathrm{M}$ ) were used for the pharmacological treatments. All of the following experiments were performed after $1 \mathrm{~h}$ treatments, unless otherwise noted.

\section{Observation of Pollen Tube Elongation and Morphology}

To measure the mean length of the pollen tubes, approximately 50 pollen tubes were detected in each of the three replicates at $0.5,1$, and $2 \mathrm{~h}$ after different treatments. The morphology of the pollen tubes was examined using a Leica DM2500 biological microscope, and digital images were captured with a Leica DFC290 digital color camera (Leica, Germany).

\section{Measurement of Cytoplasmic $\mathrm{Ca}^{2+}$ Gradient}

The pollen tubes were loaded with the fluorescent $\mathrm{Ca}^{2+}$ indicator Fluo-4/AM ester (Life Technologies, Invitrogen, USA) according to Spinelli and Gillespie (2012) with slight modifications. Briefly, after the treatments, the samples were incubated at 25 or $4^{\circ} \mathrm{C}$ in the dark in culture medium containing $20 \mu \mathrm{M}$ Fluo4/AM ester (prepared with DMSO) for $15 \mathrm{~min}$. Then, the pollen tubes were rinsed three times with the corresponding culture medium to wash out excess fluorophore. The fluorescence of at least 20 pollen tubes in each of three replicates was detected using a 488-nm argon laser attached to a Laser Scanning Confocal Microscope (LSCM, Zeiss LSM 780, Germany) with the same parameter settings, and emission signals were collected 
at $515 \mathrm{~nm}$. Image analysis was performed with pseudo color technology (Rainbow2) in ZEN 2013 software.

\section{Measurement of Cytoplasmic ROS}

The presence of ROS in the pollen tubes was assayed and visualized with CM- $\mathrm{H}_{2}$ DCF-DA (Invitrogen, USA) as described by Wilkins et al. (2011) with slight modifications. In brief, the samples were incubated in $5 \mu \mathrm{M}$ CM- $\mathrm{H}_{2}$ DCF-DA for $15 \mathrm{~min}$ in the dark; then, the excess fluorescent indicator was washed out. The specimens were mounted and photographed with a Zeiss LSM 780 LSCM (excitation at $488 \mathrm{~nm}$ and emission at $515 \mathrm{~nm}$ ). To allow comparisons between images, identical parameter settings were used throughout each experiment. The quantification of relative fluorescence units of at least 20 pollen tubes in each of three replicates was performed using the ImageJ software package, and the mean relative fluorescence intensities were calculated.

\section{Measurement of Cytoplasmic pH}

Intracellular $[\mathrm{pH}]_{\mathrm{cyt}}$ was determined in the living pollen tubes with BCECF AM (Invitrogen, USA) as described by Wilkins et al. (2015) with slight modifications. The pollen tubes were loaded with $2.5 \mu \mathrm{M}$ BCECF AM for $15 \mathrm{~min}$ followed by washing with the corresponding culture medium. The pollen tubes were only imaged within 5 to $10 \mathrm{~min}$ after the addition of BCECF AM because this time frame allowed for accurate reporting of $[\mathrm{pH}]_{\mathrm{cyt}}$. The images of at least 20 pollen tubes in each of three replicates were captured using a Zeiss LSM 780 LSCM with sequential excitation at $488 \mathrm{~nm}$ and emission at $510-550 \mathrm{~nm}$, and the image analysis was performed with pseudo color technology (Rainbow2) in ZEN 2013 software.

\section{Ultrastructure Observation with a Transmission Electron Microscope (TEM)}

A TEM analysis was performed according to Wang et al. (2014) and Sheng et al. (2006) with slight modifications. The pollen tubes were collected after treatment for $1 \mathrm{~h}$ and then fixed in $2.5 \%$ glutaraldehyde in $100 \mathrm{mM}$ PBS $(\mathrm{pH} 7.2)$ at $4^{\circ} \mathrm{C}$ for $4 \mathrm{~h}$. Then, they were washed with $100 \mathrm{mM}$ PBS and post-fixed with $2 \% \mathrm{OsO}_{4}$ for $2 \mathrm{~h}$, washed again, dehydrated in an ethanol series $(50,70,90$, and $100 \%)$ and finally embedded in Spurr's epoxy resin. Sections were cut with an LKB-V ultramicrotome, stained with $2 \%$ uranyl acetate $(\mathrm{w} / \mathrm{v})$ in $70 \%$ methanol $(\mathrm{v} / \mathrm{v})$, and $0.5 \%$ lead citrate and observed using a TEM (H-7650, Hitachi Hightechnologies Corporation, Japan) at $80 \mathrm{kV}$.

\section{Fluorescent Immunolabeling of Pectins and AGPs in the Pollen Tube Cell Wall}

The immunolabeling of pectins and AGPs in pollen tube cell walls was performed with LM19, LM20 and LM2 antibodies (PlantProbes, Leeds, UK) according to Chen et al. (2009) with slight modifications. Pollen tubes that had been treated for $1 \mathrm{~h}$ were collected and fixed in $4 \%$ paraformaldehyde in $100 \mathrm{mM}$ phosphate buffer solution (PBS, $\mathrm{pH}$ 7.2) for $30 \mathrm{~min}$ at room temperature and rinsed three times for $5 \mathrm{~min}$ each with PBS. Subsequently, the specimens were incubated for $2.5 \mathrm{~h}$ at room temperature with primary antibodies against acidic pectin (LM19), esterified pectin (LM20) and AGPs (LM2) at a dilution of 1:5. After incubation, the pollen tubes were washed with PBS three times for 10 min each, incubated with a secondary antibody, fluorescein isothiocyanate (FITC)-labeled sheep antirat IgG (KPL, Inc. USA), diluted 1:50 with PBS for at least $2 \mathrm{~h}$ at room temperature and then washed with PBS three times. The samples were mounted, and then at least 20 pollen tubes in each of three replicates were observed and photographed with a Zeiss LSM 780 LSCM (excitation at $488 \mathrm{~m}$ and emission at $522 \mathrm{~nm}$ ).

\section{Total RNA Extraction and Transcriptomic Analysis}

Pollen was pre-incubated in standard culture medium at $25^{\circ} \mathrm{C}$ in the dark for $30 \mathrm{~min}$, followed by the various treatments, including the control $\left(25^{\circ} \mathrm{C}, \mathrm{CK}\right)$, cold stress $\left(4^{\circ} \mathrm{C}, \mathrm{LT}\right)$ and NO donor $(25$ $\mu \mathrm{M}$ DEA NONOate, NO), for $1 \mathrm{~h}$ in the dark. After incubation, the pollen tubes were collected with a nylon mesh screen (200 meshes) to exclude ungerminated pollen grains; then, they were immediately subjected to grinding in liquid nitrogen. Total RNA from the pollen tubes of three independent experiments (CK1, LT1 and NO1; CK2, LT2 and NO2; and CK3, LT3 and NO3) was extracted using RNAiso Plus (TaKaRa, Japan), and the quality of the total RNA was verified using a 2100 Bioanalyzer RNA Nano chip device (Agilent, Santa Clara, CA, USA) and a NanoDrop ND-1000 spectrophotometer (NanoDrop, Wilmington, DE). The cDNA libraries were constructed and sequenced using an Illumina HiSeq ${ }^{\mathrm{TM}} 2000$ located at the Beijing Genomics Institute (Shenzhen, China; http://www.genomics.cn/index). To compare the differences in gene expression, a stringent cutoff (a probability $>0.7$ and a $\mid \log _{2}$ Ratio $\mid \geq 1.0$ ), was used to identify differentially expressed genes (DEGs).

\section{Quantitative Real-Time PCR (qRT-PCR) Analysis}

Total RNA was isolated from pollen tubes that were subjected to the various treatments described above using RNAiso Plus (TaKaRa, Japan) and treated with DNase I to remove any genomic DNA contamination. The quality of the total RNA was measured with the NanoDrop ND-1000 spectrophotometer (NanoDrop, Wilmington, DE), and the first cDNA strand was synthesized using the PrimeScript ${ }^{\mathrm{TM}}$ RT Reagent Kit with gDNA Eraser (TaKaRa, Dalian, China). The qRT-PCR was performed using SYBR ${ }^{\circledR}$ Premix Ex TaqTM II (TaKaRa, Dalian, China) on an Eppendorf Real-Time PCR System (Mastercycler ${ }^{\circledR}$ ep realplex, Germany) according to the manufacturer's protocol. The amplification regimen was set up as described by Ren et al. (2014), and three biological replicates were performed for each sample. Relative expression levels were calculated by including the C. sinensis 18 sRNA gene as the reference based on the $2^{-\Delta \Delta C T}$ method (Livak and Schmittgen, 2001). Primers used for the qRT-PCR are listed in Table S1.

\section{Statistical Analysis}

All data are expressed as the means \pm standard deviations (SD) obtained from at least three independent replicates. Statistical 
significance was calculated by one-way ANOVA using Duncan's test in the SPSS 20 software, and the significant differences among various treatment groups are represented by different letters $(P<0.05)$.

\section{RESULTS}

\section{Pollen Tube Growth and Morphological Observations}

As shown in Figure 1, cold stress and NO donor DEA NONOate significantly delayed pollen tube growth after the $0.5,1$, and 2 $h$ treatments. In addition, the NO scavenger cPTIO was used to confirm the role of NO in the cold stress-induced inhibition of pollen tube growth. Interestingly, the inhibitory effects of cold stress on pollen tube growth were markedly relieved by the simultaneous presence of cPTIO. Furthermore, under control conditions, the pollen tube showed a uniform diameter and a clear zone at the tip (Figure S1A), whereas the pollen tube exhibited obvious abnormalities such as a swollen tip and a loss of the clear zone at the tube tip after cold stress (Figure S1B) or DEA NONOate treatment for $1 \mathrm{~h}$ (Figure S1C). Moreover, the effects of cold stress on the morphology of the pollen tube tip were reduced by cPTIO (Figure S1D).

\section{Effects of Cold Stress and No on the Cytoplasmic $\mathrm{Ca}^{2+}$ Gradient in the Pollen Tube Tip}

Because $\mathrm{Ca}^{2+}$ plays a central role in the tip growth of pollen tubes, the tip-focused $\mathrm{Ca}^{2+}$ gradient is also necessary for structural organization of the cytoskeleton in angiosperm pollen tubes (Sheng et al., 2006). Pollen tubes were loaded with Fluo-4/AM to test the possible effects of cold stress and NO treatments on the $\mathrm{Ca}^{2+}$ distribution. The results showed that pollen tubes grown under normal conditions exhibited

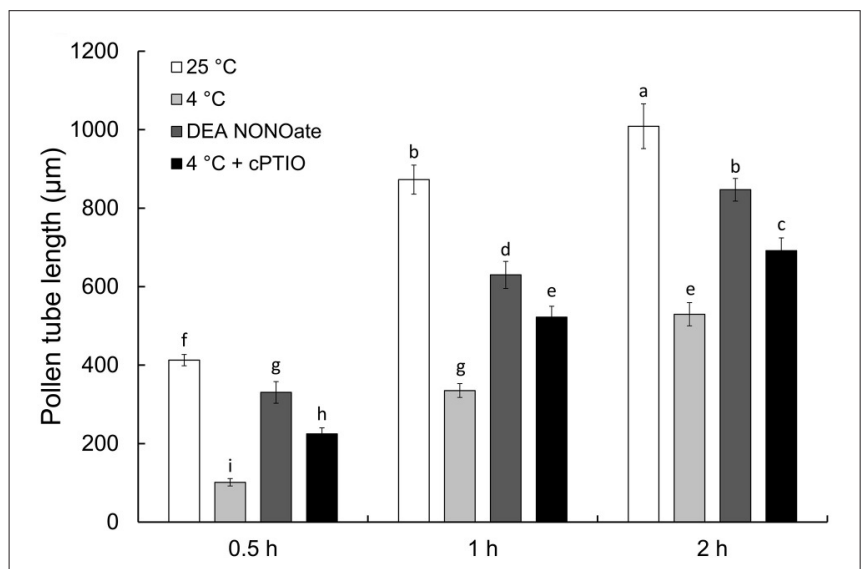

FIGURE 1 | Effects of cold stress or DEA NONOate on the growth of $\boldsymbol{C}$. sinensis pollen tube. The growth of pollen tubes was significantly delayed after treatment with cold stress $\left(4^{\circ} \mathrm{C}\right)$ or $25 \mu \mathrm{M}$ DEA NONOate, and the effects of cold stress were reduced by $200 \mu \mathrm{M}$ cPTIO. The values are the means of three replicates $\pm \operatorname{SD}(n \geq 50)$. Different letters on bars denote significant differences at $P<0.05$ according to Duncan's test. the typical tip-to-base cytoplasmic $\mathrm{Ca}^{2+}$ concentration gradient (Figures 2A,E,I), whereas this tip-focused $\mathrm{Ca}^{2+}$ gradient was disrupted after cold stress treatment, and stronger fluorescence erratically filled the entire tip of the pollen tubes (Figures 2B,F,J). Similarly, treatment with $25 \mu \mathrm{M}$ DEA NONOate also led to the disruption of the cytoplasmic $\mathrm{Ca}^{2+}$ gradient (Figures $2 \mathrm{C}, \mathbf{G}, \mathbf{K}$ ). In contrast, the disruption degree of cytoplasmic $\mathrm{Ca}^{2+}$ gradient in the pollen tubes treated with $200 \mu \mathrm{M}$ cPTIO upon cold stress was relieved (Figures 2D,H,L) compared with that in the pollen tubes treated with cold stress alone (Figures 2B,F,J).

\section{Cytoplasmic ROS is Increased in the Pollen Tube by Cold Stress and No}

To examine the relative levels of endogenous ROS, pollen tubes were labeled with $\mathrm{CM}-\mathrm{H}_{2} \mathrm{DCF}-\mathrm{DA}$, and ROS were monitored using a LSCM. In the control, the ROS fluorescence signal was distributed evenly throughout the entire pollen tube (Figures 3A,E). In comparison, the ROS fluorescence signal was significantly increased after $1 \mathrm{~h}$ of cold stress treatment, particularly in the tip region (Figures 3B,F). Similarly, a higher intensity ROS fluorescence signal was detected in the pollen tubes that were treated with $25 \mu \mathrm{M}$ DEA NONOate (Figures 3C,G) than that in the controls (Figures 3A,E). Furthermore, the ROS fluorescence signal was weaker in the pollen tubes that were treated with $200 \mu \mathrm{M}$ cPTIO under cold stress (Figures 3D,H) than in those treated with cold stress alone (Figures 3B,F), and the fluorescence signal (Figures 3D,H) was stronger than that in the controls (Figures 3A,E). Similarly, the quantification analysis showed that the average fluorescence intensity of the ROS was significantly increased by cold stress or DEA NONOate by 3.07fold and 1.86-fold compared with the control, respectively, and the increase in the average fluorescence intensity induced by cold stress was decreased 2.18-fold with $200 \mu \mathrm{M}$ cPTIO (Figure S2). These data suggest that increases in $\mathrm{NO}$ can stimulate increases in ROS under cold stress in pollen tubes of C. sinensis.

\section{Dramatic Acidification of the Pollen Tube Tip is Induced by Cold Stress and No}

Pollen tube $[\mathrm{pH}]_{\text {cyt }}$ (cytoplasmic $\mathrm{pH}$ ) has been demonstrated to play a vital role in pollen tube growth (Michard et al., 2008; Wilkins et al., 2015). We therefore investigated cold stress-induced $[\mathrm{pH}]_{\mathrm{cyt}}$ changes using the ratiometric $\mathrm{pH}$ indicator 2,7-bis-(2-carboxyethyl)-5-(and-6)-carboxy fluorescein (BCECF) acetoxymethyl ester (AM). The results showed that the $[\mathrm{pH}]_{\text {cyt }}$ decreased after $1 \mathrm{~h}$ of cold stress treatment (Figures 4B,F,J) compared to that in the control pollen tubes (Figures 4A,E,I), implying that cold stress induced the acidification in the pollen tube tip zone. Similarly, the levels of the fluorescence signal were also decreased after treatment with $25 \mu \mathrm{M}$ DEA NONOate (Figures $4 \mathbf{C}, \mathbf{G}, \mathbf{K}$ ). To examine whether $\mathrm{NO}$ is involved in cold-induced cytoplasmic acidification, $200 \mu \mathrm{M}$ cPTIO was used to treat pollen tubes under cold stress treatment, and the $[\mathrm{pH}]_{\mathrm{cyt}}$ was examined. The results showed that the degree of pollen tube cytoplasmic acidification was significantly reduced, although this reduction did not completely reverse the effects of the cold stress on the pollen 

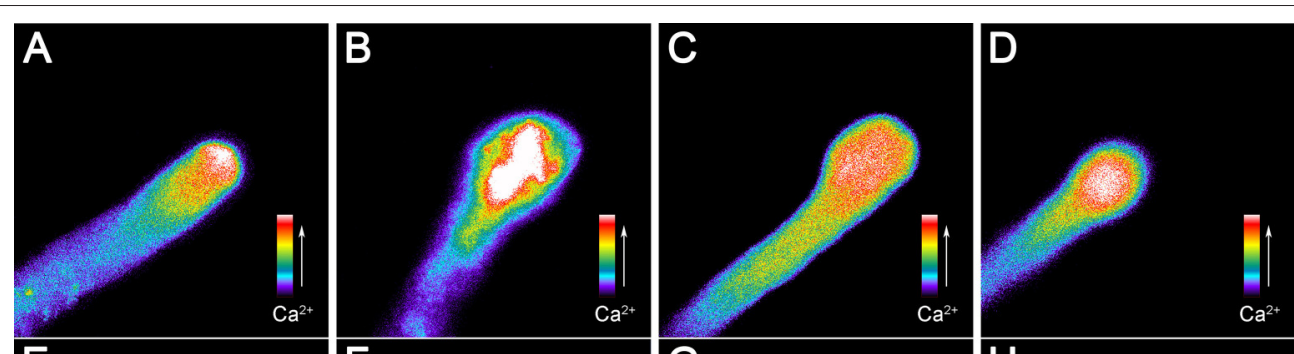

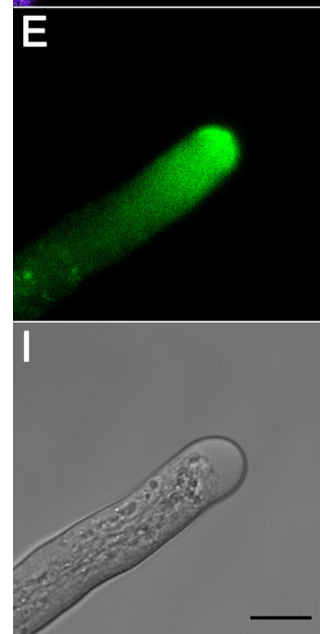

$25^{\circ} \mathrm{C}$

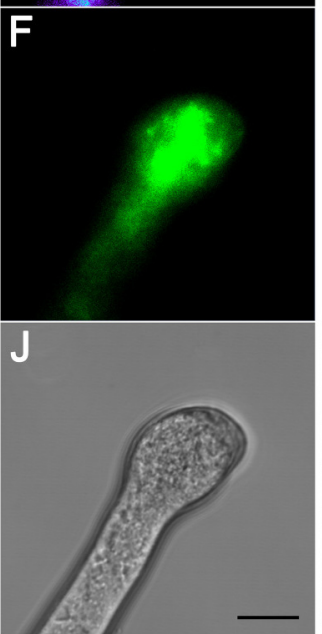

$4{ }^{\circ} \mathrm{C}$

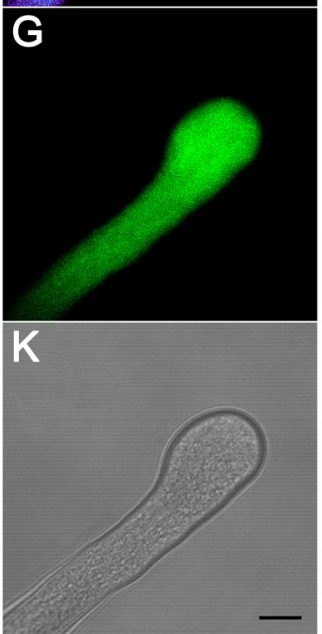

DEA NONOate

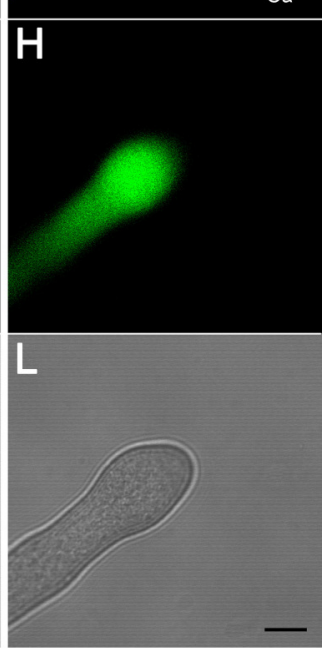

$4{ }^{\circ} \mathrm{C}+\mathrm{CPTIO}$

FIGURE 2 | Changes in the tip-focused $\mathbf{C a}^{2+}$ gradient in C. sinensis pollen tubes after treatment with cold stress or DEA NONOate. Pollen tubes were loaded with the fluorescent $\mathrm{Ca}^{2+}$ indicator Fluo-4/AM ester, and the fluorescence was detected using LSCM (488 nm excitation and 515 emission). The control pollen tubes exhibited the typical tip-focused cytoplasmic $\mathrm{Ca}^{2+}$ concentration gradient (A). The corresponding fluorescent image (E) and bright field image (I) are shown below. The pollen tubes that were treated with cold stress (B) or $25 \mu \mathrm{M}$ DEA NONOate (C) showed the disrupted cytoplasmic Ca ${ }^{2+}$ gradient. The corresponding fluorescent images $(\mathbf{F}, \mathbf{G})$ and bright field images $\mathbf{( J , K )}$ are shown below. The effects of cold stress on the cytoplasmic Ca ${ }^{2+}$ gradient were weakened by $200 \mu \mathbf{M}$ CPTIO (D). The corresponding fluorescent image (H) and bright field image (L) are shown. At least 20 pollen tubes were observed and photographed in each of three replicates, and one representative image per treatment was displayed. Bar $=10 \mu \mathrm{m}$.

tube $[\mathrm{pH}]_{\mathrm{cyt}}$ (Figures $\left.4 \mathrm{D}, \mathbf{H}, \mathbf{L}\right)$. These data show the functional importance of NO in the process of cold stress-induced pollen tube cytoplasmic acidification and provide insight into the mechanisms of NO involved in cold-inhibited pollen tube growth.

\section{Cold Stress and No Disrupt the Distribution of Organelles and Induce Cell Wall \\ Abnormalities in C. sinensis Pollen Tubes}

TEM was performed, and the results showed that the extreme apical zone of the pollen tube was filled with numerous secretory vesicles in the control condition (Figures 5A,E). Fusion of the vesicles with the plasma membrane was frequently observed, as shown by the black arrows (Figure 5E), indicating that the cell wall materials were actively released into the cell wall. A large number of other organelles, particularly mitochondria and smooth endoplasmic reticulum (sER), accumulated in the subapical zone (Figures 5A,E). However, substantial variation was observed in the pollen tube tips that were treated with cold stress (Figures 5B,F) or exogenous $\mathrm{NO}$ for $1 \mathrm{~h}$ (Figures 5C,G). The most obvious change was a disruption of the distribution of organelles, as shown by the sharp decline in the number of vesicles, mitochondria and sER, and the feature of other organelles, including the rough endoplasmic reticulum (rER) and vacuoles at the tip of the pollen tube (Figures 5B,C,F,G). Moreover, the configuration of the rER was altered, and it appeared to wrap around vacuoles and other organelles when treated with cold stress (Figure S3A) or exogenous NO (Figure S3B). Furthermore, the use of cPTIO only partly reversed the effect of cold stress on the organelle ultrastructure of the pollen tubes (Figures 5D,H), suggesting that $\mathrm{NO}$ is not a unique factor in the process in which cold stress affects the organelle ultrastructure of pollen tubes.

In the control pollen tubes, thick, brown, smooth cell walls were attached at the base of the pollen tube (Figures S4A,B) to maintain the mechanical support, whereas the cell wall at the tip region was thin, translucent and bumpy to maintain the high flexibility of the tip region (Figures 5A,E); these differences in the cell wall resulted in fast polarized growth of the pollen tube. However, the typical feature of the cell wall at the tip region was changed to that of a cell wall at the base region after the cold stress treatment (Figures 5B,F) or treatment with $25 \mu \mathrm{M}$ DEA 


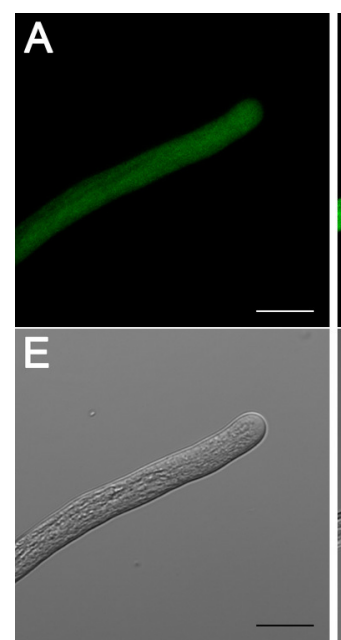

$25^{\circ} \mathrm{C}$
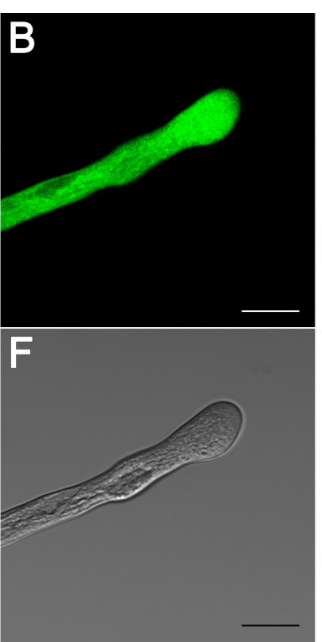

$4{ }^{\circ} \mathrm{C}$
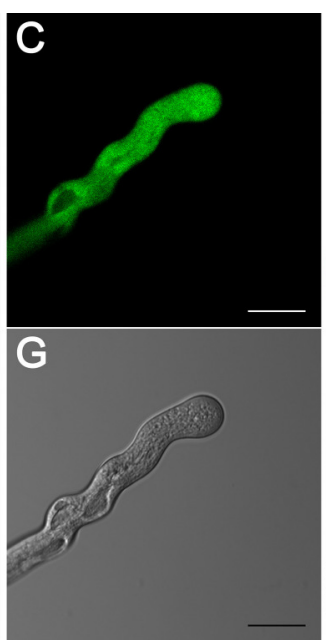

DEA NONOate
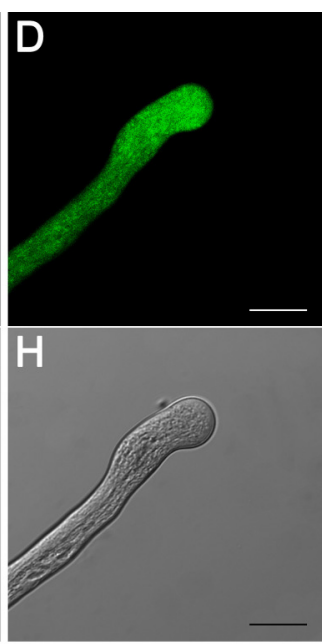

$4{ }^{\circ} \mathrm{C}+\mathrm{CPTIO}$

FIGURE 3 | Effects of cold stress or DEA NONOate on cytoplasmic ROS in C. sinensis pollen tubes. In the control pollen tubes, the ROS fluorescence signal was symmetrical and weak and was distributed throughout almost the entire pollen tube (A). After treatment with cold stress (B) or $25 \mu \mathrm{M}$ DEA NONOate (C) for 1 h, the ROS fluorescence signal significantly increased, particularly in the tip region. The increase in the ROS fluorescence signal stimulated by cold stress was largely reduced after treatment with $200 \mu \mathrm{M}$ cPTIO (D). The corresponding bright field images are shown below (E-H). At least 20 pollen tubes were observed and photographed in each of three replicates, and one representative image per treatment was displayed. Bar $=20 \mu \mathrm{m}$.
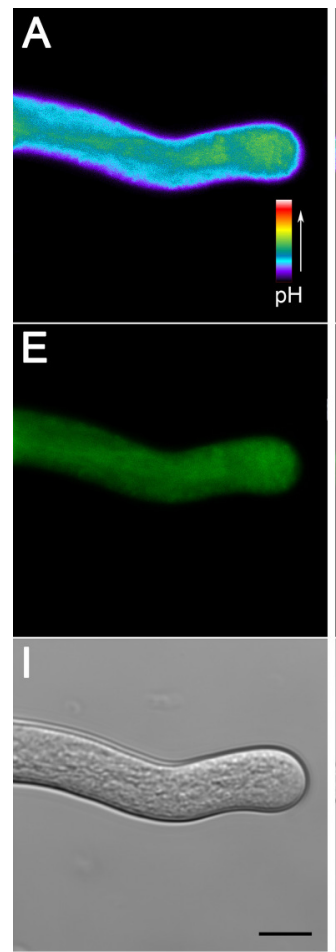

$25^{\circ} \mathrm{C}$
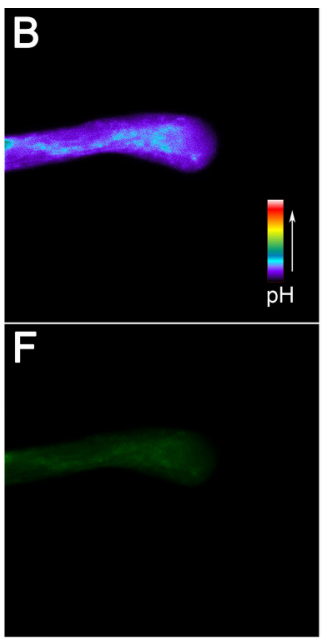

J

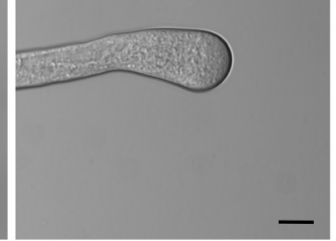

$4{ }^{\circ} \mathrm{C}$
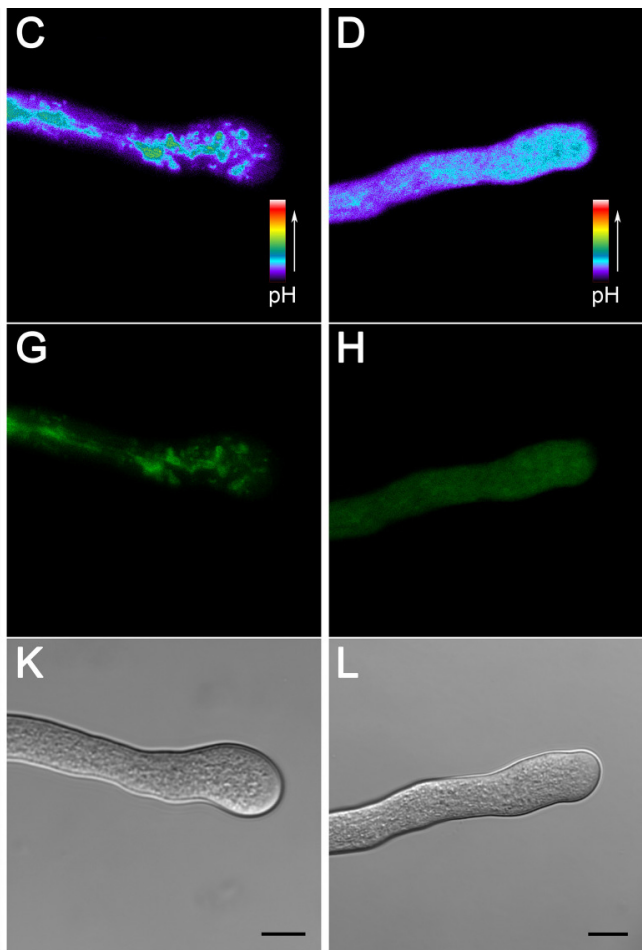

DEA NONOate

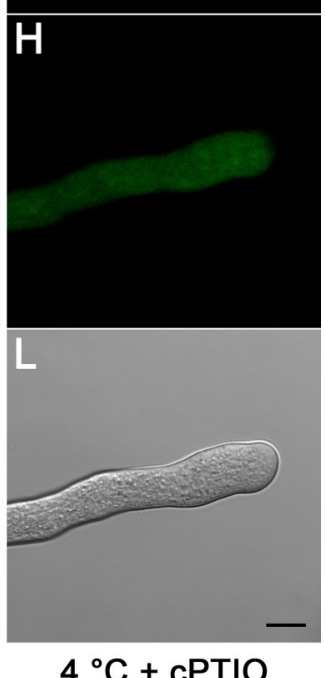

FIGURE 4 | Cold stress or DEA NONOate induces cytoplasmic $\mathbf{p H}\left([\mathbf{p H}]_{\text {cyt }}\right.$ ) acidification in $\mathbf{C}$. sinensis pollen tubes. Pollen tubes $[\mathrm{pH}]_{\mathrm{cyt}}$ were labeled with the $\mathrm{pH}$ indicator BCECF AM prior to imaging using LSCM. Compared with the control pollen tubes (A), the levels of the fluorescence signal were significantly decreased after $1 \mathrm{~h}$ of cold stress $\mathbf{( B )}$ or $25 \mu \mathrm{M}$ DEA NONOate (C) treatments, and the degree of pollen tube cytoplasmic acidification mediated by cold stress was significantly reduced after treatment with $200 \mu \mathrm{M}$ cPTIO (D). The corresponding fluorescent images (E-H) and bright field images (I-L) are shown below, respectively. At least 20 pollen tubes were observed and photographed in each of three replicates, and one representative image per treatment was displayed. Bar $=10 \mu \mathrm{m}$. 


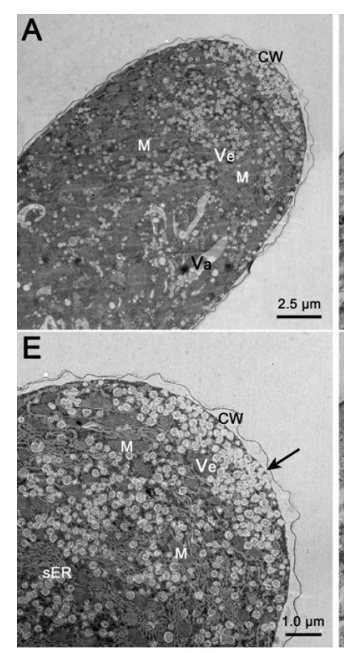

$25^{\circ} \mathrm{C}$
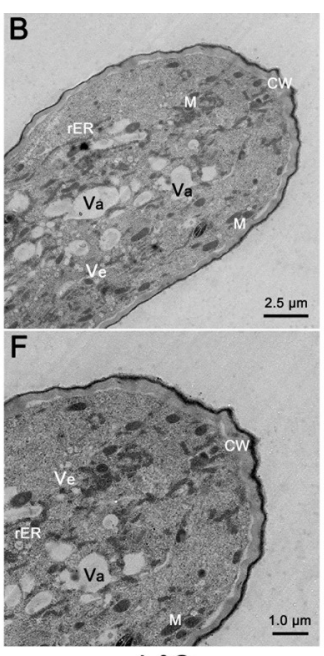

$4{ }^{\circ} \mathrm{C}$
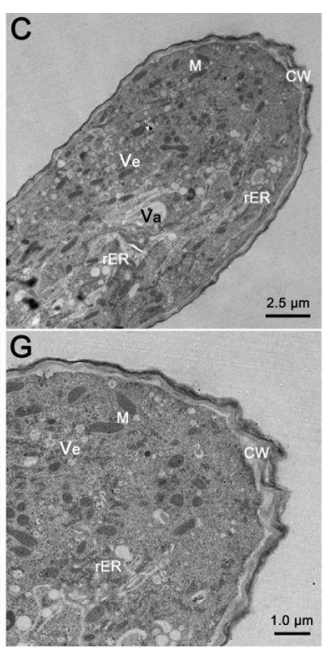

DEA NONOate
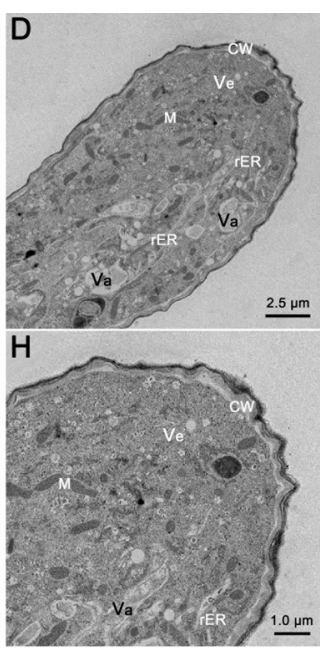

$4{ }^{\circ} \mathrm{C}+\mathrm{CPTIO}$

FIGURE 5 | Electron micrographs of $\boldsymbol{C}$. sinensis pollen tubes after $\mathbf{1} \mathbf{h}$ of cold stress or DEA NONOate treatment. The extreme apical zone of the control pollen tube was filled with numerous secretory vesicles (Ve), and a large number of other organelles, particularly mitochondria (M) and smooth endoplasmic reticulum (sER), and wrapped in a thin, translucent, bumpy cell wall $(\mathbf{A}, \mathbf{E})$. The black arrows indicate the fusion of vesicles with the plasma membrane (E). The polarized distribution of vesicles, mitochondria and sER was disrupted, and rough endoplasmic reticulum (rER) and vacuoles (Va) existed in the tip region; the morphology of the cell wall (CW) was changed by the cold stress (B,F) or $25 \mu \mathrm{M}$ DEA NONOate treatment (C,G). Treatment with $200 \mu \mathrm{M}$ cPTIO effectively reduced the effects of cold stress on the cell wall ultrastructure, but there were negligible changes in the distribution and ultrastructure of the organelles (D,H). M, mitochondria; Ve, vesicle; Va, vacuole; CW, cell wall.

NONOate (Figures 5C,G). In addition, treatment with $200 \mu \mathrm{M}$ cPTIO effectively reduced the effects of cold stress on the cell wall ultrastructure (Figures 5D,H), indicating that the cell wall was another key factor in the process of NO involvement in coldinhibited C. sinensis pollen tube growth. Thus, we hypothesized that the cold-induced NO disrupts the organization of the cell wall, resulting in the retarded growth of the tubes and tip swelling.

\section{Effects of Cold Stress and No Treatment on the Distribution of Pectin and AGPs}

To further confirm the role of the cell wall, we studied the distribution of the cell wall components in pollen tubes. In pollen tubes grown under standard conditions, the localization of LM19-reactive pectin indicates the distribution of acidic pectin only in the basal regions of the tube, and the intensity of the antigen signal decreases gradually toward the apex of the tube (Figure 6A), whereas the localization of the LM20-reactive esterified pectin was limited to the very tip of the growing tubes (Figure 6E). In contrast, pollen tubes treated with cold stress (Figures 6B,F) or $25 \mu \mathrm{M}$ DEA NONOate (Figures 6C,G) showed completely different pectin distributions compared with the control pollen tubes (Figures 6A,E); for example, acidic pectins were detected across the entire surface of the pollen tubes, including the tips (Figures 6B,C), and esterified pectins were detected only in the basal region near the germinating aperture (Figures 6F,G). Notably, the fluorescence signal of acidic pectin at the pollen tube tip was decreased by $200 \mu \mathrm{M}$ cPTIO under cold stress (Figure 6D). In addition, treatment with cPTIO increased the distribution of esterified pectin on the shank of the pollen tubes, although the signal was still not detected at the pollen tube tip (Figure $\mathbf{6 H}$ ). Furthermore, pollen tubes grown under standard conditions showed a characteristic dotstrengthening with remarkable periodicity of AGPs deposition along the entire length, as revealed by immune-localization with the LM2 antibodies, and the ring-like structures based on dotstrengthening were visualized, particularly in the apical region of the pollen tubes (Figure 7A) In contrast, the pollen tubes that were treated with cold stress or $25 \mu \mathrm{M}$ DEA NONOate showed a completely different distribution of AGPs compared to the control. The dot-strengthening feature and the ring-like structures at the tip disappeared, and the fluorescence signal was observed only on the shank region of the pollen tubes (Figures 7B,C). However, the changes in the AGPs distribution caused by cold stress were effectively reversed by $200 \mu \mathrm{M} \mathrm{cPTIO}$ (Figure 7D).

\section{Signaling Pathway Related DEGs Quantified and Identified from Cold Stress or No Treatments}

Differentially expressed genes (DEGs) were identified according to Zhao et al. (2015), with a probability $>0.7$ and an estimated absolute $\mid \log _{2}$ Ratio $\mid \geq 1.0$. Comparing the CK library (CK1, CK2, and CK3) with the LT library (LT1, LT2, and LT3), scilicet CK-VS-LT, there were 278 signaling pathway-related DEGs (130 genes up-regulated and 148 genes down-regulated, 130/148), among which there were 12 genes associated with $\mathrm{NO}$ synthesis and $42,80,11,25,46,28$, and 25 genes related to cGMP, $\mathrm{Ca}^{2+}$, ROS, $\mathrm{pH}$, actin, the cell wall, and the MAPK cascade, respectively (Figure 8A, Table S2). Similarly, 221 signaling pathway-related 

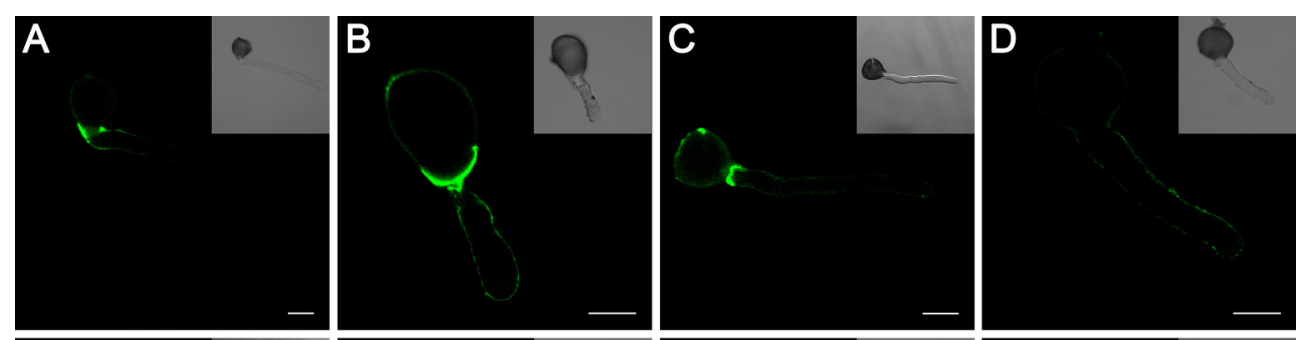

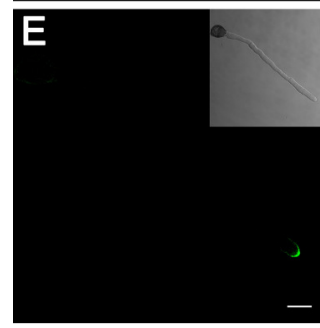

$25^{\circ} \mathrm{C}$

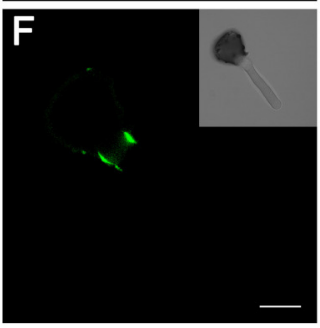

$4{ }^{\circ} \mathrm{C}$

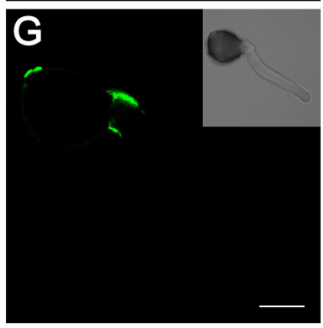

DEA NONOate

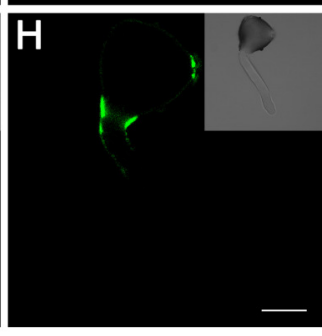

$4{ }^{\circ} \mathrm{C}+\mathrm{cPTIO}$

FIGURE 6 | Effects of cold stress and DEA NONOate on the distribution of acidic pectins and esterified pectins in the pollen tube cell wall of $\boldsymbol{C}$. sinensis. LM19 labeling of the control pollen tubes observed by LSCM showed that strong fluorescence occurred in the basal site of the tube wall and decreased gradually toward the tip region of the pollen tube $\mathbf{( A )}$, but fluorescence occurred along the entire pollen tube wall, including the tip region, in the pollen tubes treated with cold stress (B), or $25 \mu \mathrm{M}$ DEA NONOate (C). The fluorescence signal of the LM19 labeled acidic pectins at the pollen tube tip was decreased by $200 \mu \mathrm{M}$ cPTIO under cold stress (D). LM20 labeling of the control pollen tubes observed by LSCM showed that the esterified pectins localized to the tip region of the pollen tubes (E). LM20 labeling of the pollen tubes treated with cold stress or $25 \mu \mathrm{M}$ DEA NONOate observed by LSCM showed that the esterified pectins accumulated only in the basal region near the germinating aperture $(\mathbf{F}, \mathbf{G})$. Treatment with $200 \mu \mathrm{M}$ cPTIO increased the distribution of esterified pectins on the shank of the pollen tubes but was still not detected in the pollen tube tip region $\mathbf{( H )}$. Corresponding bright field images are shown at a reduced size. At least 20 pollen tubes were observed and photographed in each of three replicates, and one representative image per treatment was displayed. Bar $=20 \mu \mathrm{m}$.

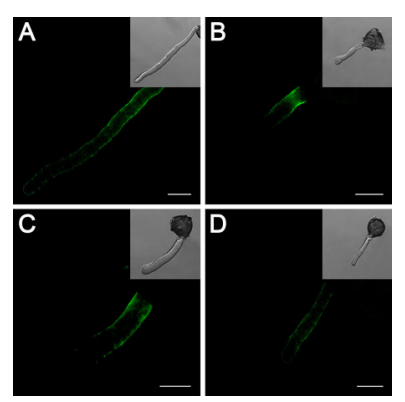

FIGURE 7 | Effects of cold stress and DEA NONOate on the distribution of AGPs in C. sinensis pollen tubes. Pollen tubes incubated under standard conditions exhibited a characteristic dot-strengthening with remarkable periodicity of AGPs deposition along the entire length, as shown by immunolocalization with the LM2 antibodies, and the ring-like structures based on dot-strengthening were visualized in the apical region of the pollen tubes (A). After treatment with cold stress or $25 \mu \mathrm{M}$ DEA NONOate, the AGPs distribution showed no dot-strengthened and ring-like structures, and the fluorescence signal was deposited only on the shank region of the pollen tubes $\mathbf{( B , C )}$. The fluorescence signal of the AGPs occurred along the entire pollen tube wall, including the tip region, under cold stress after $200 \mu \mathrm{M}$ CPTIO treatment (D). Corresponding bright field images are shown at a reduced size. At least 20 pollen tubes were observed and photographed in each of three replicates, and one representative image per treatment was displayed. Bar = $20 \mu \mathrm{m}$.

DEGs (117/104) were detected in CK-VS-NO, and the numbers of DEGs involved in NO synthesis, cGMP, $\mathrm{Ca}^{2+}$, ROS, $\mathrm{pH}$, actin, the cell wall, and the MAPK cascade were as follows:
$15,36,64,6,17,48,18$, and 17, respectively (Figure 8B, Table S3). In addition, more DEGs were involved in $\mathrm{Ca}^{2+}$ signaling than other signaling-related DEGs that were identified in the treatments, indicating the importance of $\mathrm{Ca}^{2+}$ in the process of the pollen tube response to cold stress and $\mathrm{NO}$ treatments.

In this study, hundreds of signaling pathway-related DEGs involved in the signal transduction pathways responding to cold stress (CK-VS-LT) and exogenous NO treatments (CKVS-NO) were identified, and 89 genes were co-expressed in CK-VS-LT and CK-VS-NO (Figure S5A), including $5 \mathrm{NO}$ synthesis-related genes, 16 cGMP-related genes, $21 \mathrm{Ca}^{2+}$. related genes, 3 ROS-related genes, 9 pH-related genes, 20 actin-related genes, 7 cell wall-related genes, and 8 MAPK cascade-related genes (Table 1). Among these genes, some genes that were closely related to pollen tube polarized growth were detected, such as cyclic nucleotide-gated ion channels (CNGCs, Unigene4594_All and Unigene8255_All), Rac-like GTP-binding protein (RACs, CL1173.Contig1_All, and Unigene4635_All), actin-depolymerizing factor (ADF, Unigene20449_All), callose synthase (Unigene17733_All), pectin methyl esterase (PME, Unigene4524_All and Unigene12294_All), and glutamate receptor (GLRs, CL4694.Contig1_All, and CL6126.Contig4_All). Interestingly, $\mathrm{Ca}^{2+}$ signaling related genes were the most common, and they accounted for $23.60 \%$ of all co-expressed genes (Figure $\mathrm{S} 5 \mathrm{~B}$ ), which further confirmed that $\mathrm{Ca}^{2+}$ plays an important role in the process of the pollen tube response to cold stress. 


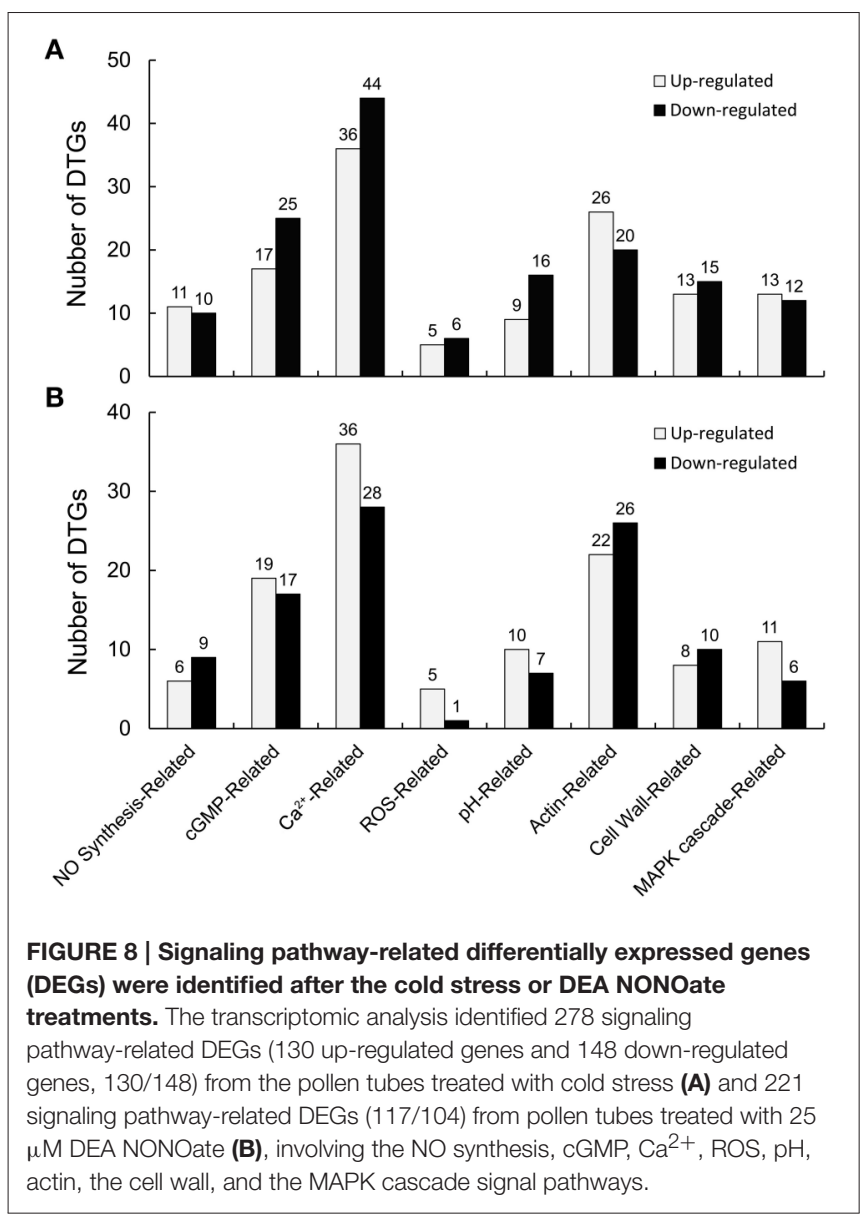

\section{Quantitative Real-Time PCR (qRT-PCR) Analysis}

To validate the expression profiles of the genes in our Illumina RNA-Seq results and to further verify the key functions of $\mathrm{NO}$ in the pollen tube response to cold stress, the expression levels of 22 critical DEGs were analyzed using qRT-PCR after treatment with cold stress $\left(4^{\circ} \mathrm{C}\right), 25 \mu \mathrm{M}$ DEA NONOate or $4^{\circ} \mathrm{C}$ and $200 \mu \mathrm{M}$ cPTIO. These genes encode the following: NOassociated protein 1 (NOA1), spermine synthase, cytochrome P450 93A1-like (P450), protein tyrosine phosphatase (PTP), two pore calcium channel protein 1-like (TPC1), cyclic nucleotidegated ion channels (CNGCs), calcium-dependent protein kinase SK5 (CDPK5), mitogen-activated protein kinase kinase kinase (MAP3K), WRKY transcription factors (WRKYs), Rac-like GTPbinding proteins (RACs), glutamate receptor 2.7-like (GLR2.7), NADPH oxidase (NOX), $\mathrm{Na}^{+} / \mathrm{H}^{+}$antiporter, plasma membrane $\mathrm{H}^{+}$-ATPase $\left(\mathrm{PM} \mathrm{H}^{+}\right.$-ATPase), PME, and actin-depolymerizing factor 1 (ADF1). As shown in Figure 9, the expression tendency of these genes exhibited a close similarity to the Illumina RNASeq results (Table 1). In addition, the changes in the expression levels of most genes were relieved by $200 \mu \mathrm{M}$ cPTIO treatment under cold stress, such as PTP, TPC1, CNGC1, CDPK5, RACs, GLR2.7, $\mathrm{NOX}, \mathrm{Na}^{+} / \mathrm{H}^{+}$antiporter, PME1, and ADF1, indicating that these genes participated in the pollen tube response to cold stress, possibly through the regulation of the NO signaling pathway.

In our previous study, proline (Pro) accumulation played an important role in the process of $\mathrm{NO}$ involvement in coldinhibited C. sinensis pollen tube growth (Wang et al., 2012), but the mechanism of Pro accumulation remains unclear. Here, the expression of three rate-limiting enzyme genes in Pro metabolism, including $\Delta^{1}$-pyrroline-5-carboxylate synthetase (P5CS), ornithine- $\delta$-aminotransferase $(\delta-O A T)$ and proline dehydrogenase (ProDH), were detected by qRT-PCR (Figure 10). The results showed that the expression of CsP5CS increased after cold stress treatment but did not change after treatment with $25 \mu \mathrm{M}$ DEA NONOate. Additionally, the expression of Cs $\delta$ $O A T$ was induced by cold stress or exogenous NO, and the induction that resulted from cold stress was significantly reduced by $200 \mu \mathrm{M}$ cPTIO. Interestingly, exogenous NO decreased the expression of $\mathrm{Cs} P$ roDH, and $\mathrm{CPTIO}$ caused a greater increase in CsProDH expression under cold stress compared with cold stresstreated pollen tubes. These results suggest that NO regulated Pro accumulation by increasing the expression of $C s \delta-O A T$ instead of CsP5CS and by reducing the expression of CsProDH in C. sinensis pollen tubes responding to cold stress.

\section{DISCUSSION}

Research over the last few decades has identified NO as an important signaling molecule with diverse biological functions in plants. NO plays a crucial role in growth and development, from germination to senescence, and is also involved in plant responses to biotic and abiotic stresses, including cold stress (Sehrawat et al., 2013b). In addition, previous investigations have demonstrated that NO is involved in the regulation of pollen tube growth, particularly in the polarized tip (Prado et al., 2004). Our data show that both $\mathrm{NO}$ and cold stress inhibit $C$. sinensis pollen tube growth and lead to tip morphological abnormalities and that the NO scavenger cPTIO is able to effectively mitigate the effects of cold stress on pollen tubes, implying that NO participates in the process of cold stress-inhibited $C$. sinensis pollen tube growth (Wang et al., 2012). This is consistent with the results of (Prado et al., 2004, 2008) who reported NO as a negative regulator of pollen tube growth in Lilium longiorum and Arabidopsis thaliana.

It is well known that NO production is mainly mediated through three NO synthases (NOS) with different localizations and functions in animals, which catalyze the conversion of LArg to L-citrulline and NO (Qiao and Fan, 2008). However, the pathways for producing NO in plant tissues are complicated, diverse and undefined and remain a matter of discussion. Current studies have revealed that NOS activity has also been detected in higher plants, although no direct gene coding for a canonical NOS protein has been found in the genomes of Arabidopsis or any other higher plants (Domingos et al., 2015). For example, Zhao et al. (2007) reported that NOS-dependent NO production was associated with salt tolerance in Arabidopsis, and NOS-like activity-dependent endogenous NO production enhanced the tolerance to cold stress in Chorispora bungeana suspension culture cells (Liu et al., 2010). In our previous report, 
TABLE 1 | Co-expressed DEGs in CK-VS-LT and CK-VS-NO involved in NO signaling pathway under cold stress.

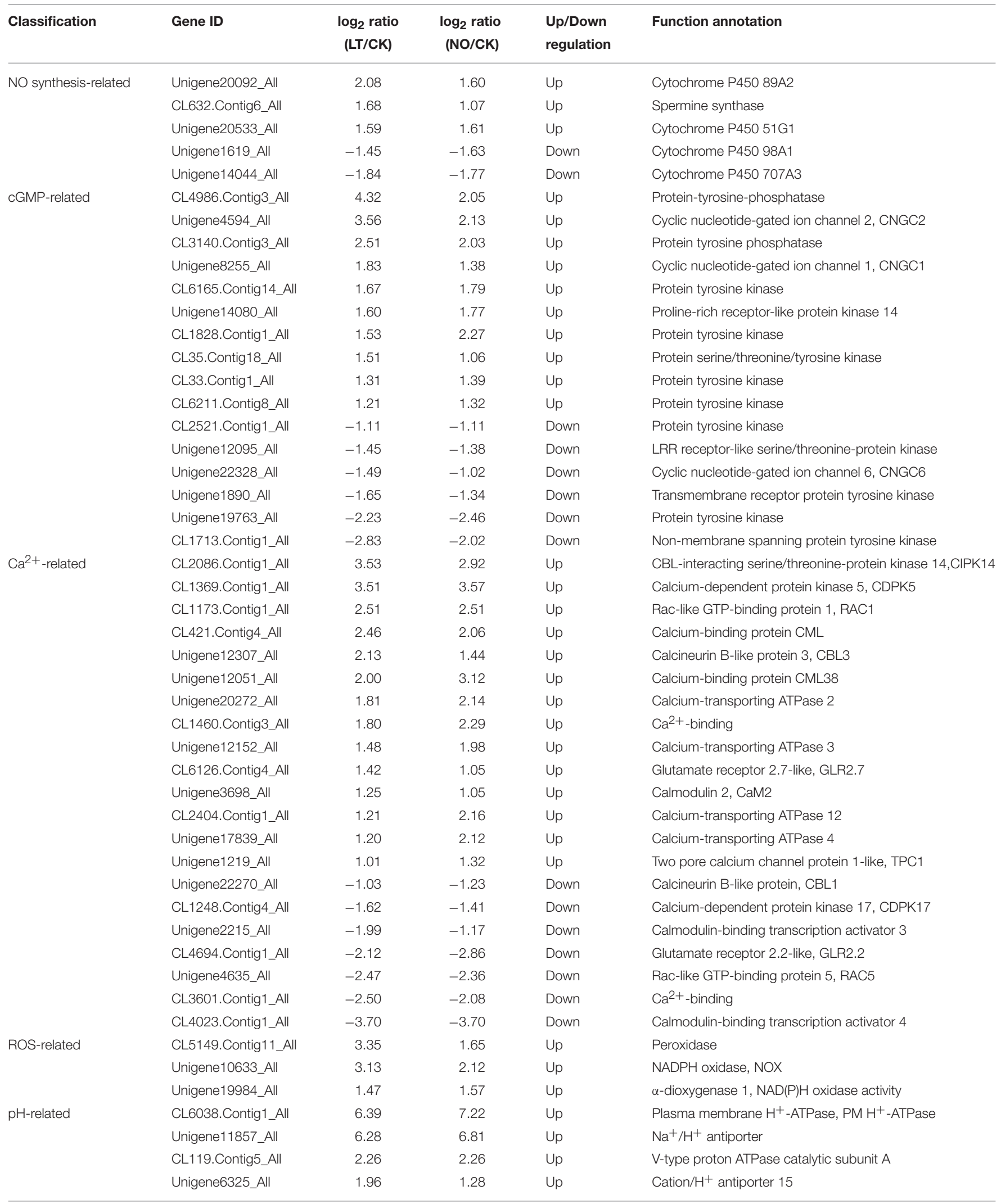


TABLE 1 | Continued

\begin{tabular}{|c|c|c|c|c|c|}
\hline Classification & Gene ID & $\begin{array}{c}\log _{2} \text { ratio } \\
\text { (LT/CK) }\end{array}$ & $\begin{array}{r}\log _{2} \text { ratio } \\
(\mathrm{NO} / \mathrm{CK})\end{array}$ & $\begin{array}{l}\text { Up/Down } \\
\text { regulation }\end{array}$ & Function annotation \\
\hline & CL287.Contig3_All & 1.36 & 1.28 & Up & Cation/ $\mathrm{H}^{+}$antiporter 15 \\
\hline & CL853.Contig4_All & 1.26 & 1.01 & Up & Inorganic pyrophosphatase \\
\hline & CL402.Contig21_All & 1.09 & 1.46 & Up & Cation/ $/ \mathrm{H}^{+}$antiporter 15 \\
\hline & CL332.Contig46_All & -1.50 & -1.50 & Down & Vacuolar proton translocating ATPase 100 kDa subunit \\
\hline & Unigene8798_All & -2.48 & -1.13 & Down & Cation/H+ antiporter 15-like \\
\hline \multirow[t]{20}{*}{ Actin-related } & Unigene15928_All & 5.18 & 6.72 & Up & Actin \\
\hline & Unigene6231_All & 4.55 & 6.95 & Up & Actin-binding \\
\hline & CL2575.Contig3_All & 3.92 & 2.48 & Up & Formin-like protein 3 \\
\hline & Unigene3512_All & 3.67 & 3.21 & Up & Myosin- $\mathrm{H}$ heavy chain-like \\
\hline & Unigene20449_All & 2.78 & 2.86 & Up & Actin-depolymerizing factor 1, ADF1 \\
\hline & Unigene20955_All & 2.27 & 2.36 & Up & Actin cytoskeleton organization \\
\hline & CL1355.Contig4_All & 1.95 & 2.84 & Up & Myosin-J heavy chain-like \\
\hline & CL3551.Contig2_All & 1.84 & 2.47 & Up & Kinesin-1 \\
\hline & Unigene1957_All & 1.83 & 1.86 & Up & Kinesin family member 2/24 \\
\hline & CL274.Contig4_All & 1.72 & 1.43 & Up & Caltractin ( $\mathrm{Ca}^{2+}$-binding protein) \\
\hline & Unigene2298_All & 1.30 & 1.34 & Up & Myosin-Vb-like \\
\hline & Unigene19227_All & 1.07 & 1.01 & Up & Actin nucleation \\
\hline & CL4715.Contig1_All & 1.00 & 1.91 & Up & Positive regulation of actin nucleation \\
\hline & Unigene15770_All & -1.06 & -1.01 & Down & Actin-related protein 4 \\
\hline & Unigene8236_All & -1.12 & -1.43 & Down & 65-kDa microtubule-associated protein 3 \\
\hline & Unigene22115_All & -1.13 & -1.35 & Down & Villin-1 \\
\hline & Unigene22529_All & -1.55 & -2.94 & Down & Formin-like protein 7 \\
\hline & CL3197.Contig7_All & -1.58 & -2.30 & Down & Formin-like protein 20 \\
\hline & Unigene2909_All & -2.78 & -1.19 & Down & Myosin-Vb-like \\
\hline & CL797.Contig2_All & -4.13 & -2.00 & Down & F-actin-capping protein subunit $\alpha$ \\
\hline \multirow[t]{7}{*}{ Cell wall-related } & CL5329.Contig2_All & 2.31 & 1.96 & Up & $\beta$-1,3-galactosyltransferase 20 \\
\hline & Unigene4524_All & 2.12 & 1.68 & Up & Pectin methyl esterase 2, PME2 \\
\hline & Unigene17733_All & 2.04 & 1.09 & Up & Callose synthase 12 \\
\hline & Unigene12294_All & 1.82 & 1.74 & Up & Pectin methyl esterase 1, PME1 \\
\hline & CL240.Contig9_All & -1.33 & -2.53 & Down & Pollen Ole e1 allergen and extensin family protein \\
\hline & Unigene11072_All & -1.50 & -1.35 & Down & Arabinogalactan peptide 22, AGP22 \\
\hline & CL1401.Contig2_All & -2.65 & -1.18 & Down & Probable pectinesterase/pectinesterase inhibitor 21 \\
\hline \multirow[t]{8}{*}{ MAPK cascade-related } & Unigene20102_All & 1.87 & 1.05 & Up & Mitogen-activated protein kinase kinase kinase 3, MAP3K3 \\
\hline & Unigene3464_All & 1.50 & 1.69 & Up & Activation of MAPKK activity \\
\hline & Unigene18265_All & 1.50 & 1.49 & Up & WRKY transcription factor 21 \\
\hline & Unigene4667_All & 1.43 & 1.04 & Up & Mitogen-activated protein kinase kinase kinase, MAP3K \\
\hline & CL580.Contig48_All & 1.27 & 1.07 & Up & Protein kinase and PP2C-like domain-containing protein \\
\hline & Unigene18965_All & 1.24 & 1.32 & Up & Probable CCR4-associated factor 1 homolog 11 \\
\hline & Unigene21639_All & -1.27 & -1.83 & Down & WRKY transcription factor 19 \\
\hline & Unigene7269_All & -1.60 & -1.05 & Down & WRKY transcription factor 7 \\
\hline
\end{tabular}

NOS-like activity was confirmed to participate in the coldinduced NO production in C. sinensis pollen tubes (Wang et al., 2012). In the present study, the expression of the NO-associated protein 1 gene (NOA1, Unigene17147_All) was induced by cold stress, and cPTIO increased the effects of cold stress (Figure 9), which further supports the conclusion about cold stress-induced NO production partly from NOS-like activity in our previous studies. In addition to NOS-mediated NO production, several other NO biosynthetic enzymes may function in plant cells, including the $\mathrm{NAD}(\mathrm{P}) \mathrm{H}$-dependent nitrate reductase (NR), xanthine oxidase/dehydrogenase and cytochrome P450 (Zhao et al., 2009; Sanz et al., 2015). Many potential NO productionrelated genes were also identified by transcriptomic analyses in the process of the C. sinensis pollen tube response to cold stress, such as the cytochrome P450 family genes and arginine metabolism-related genes (Table S3). Interestingly, NR genes were not among the differentially expressed genes after treatment with cold stress, although the role of NR in the cold-induced NO accumulation in C. sinensis pollen tubes cannot be excluded. These results indicate that the sources of NO production under 

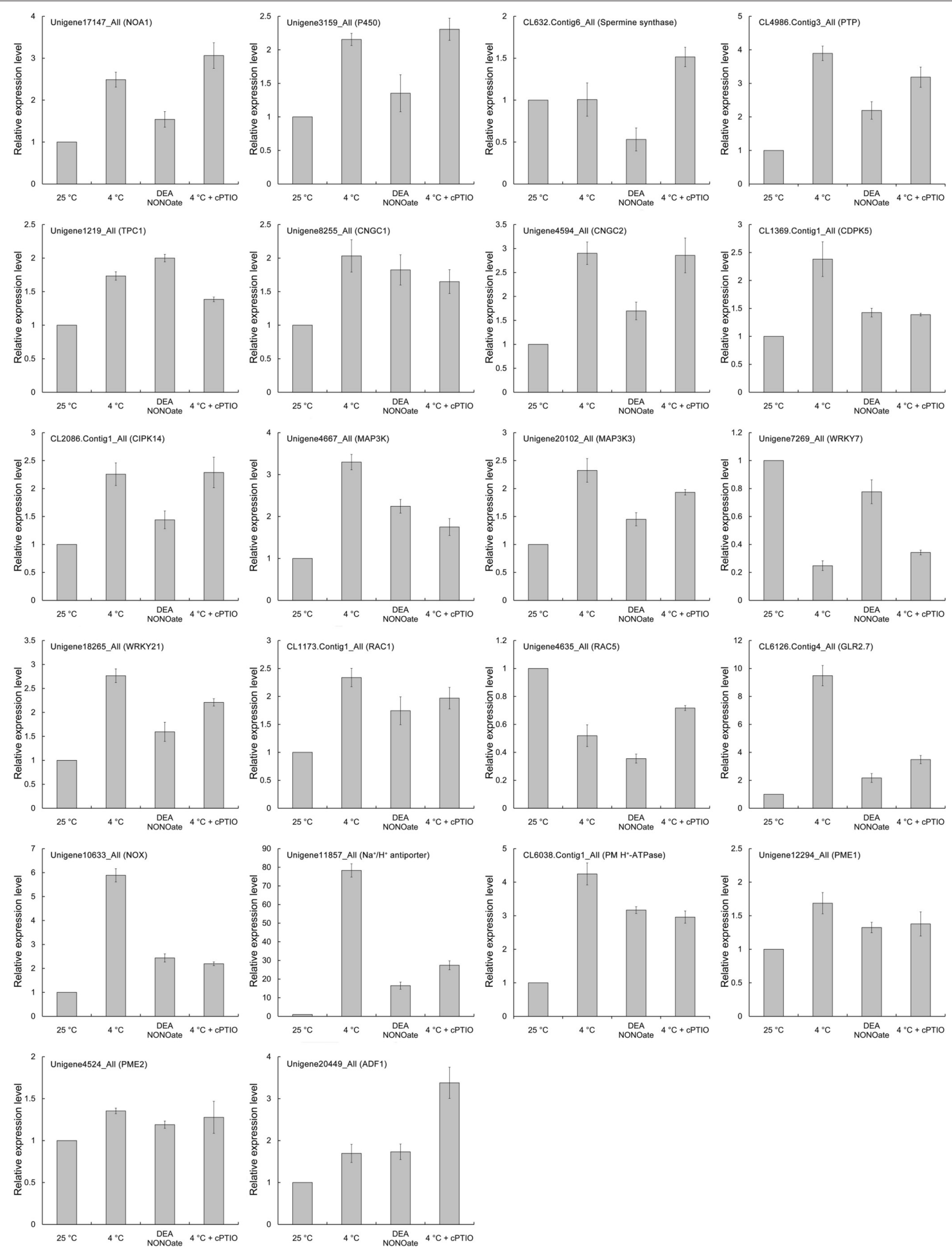

FIGURE 9 | QRT-PCR validation for 22 co-expressed DEGs in CK-VS-LT and CK-VS-NO. 


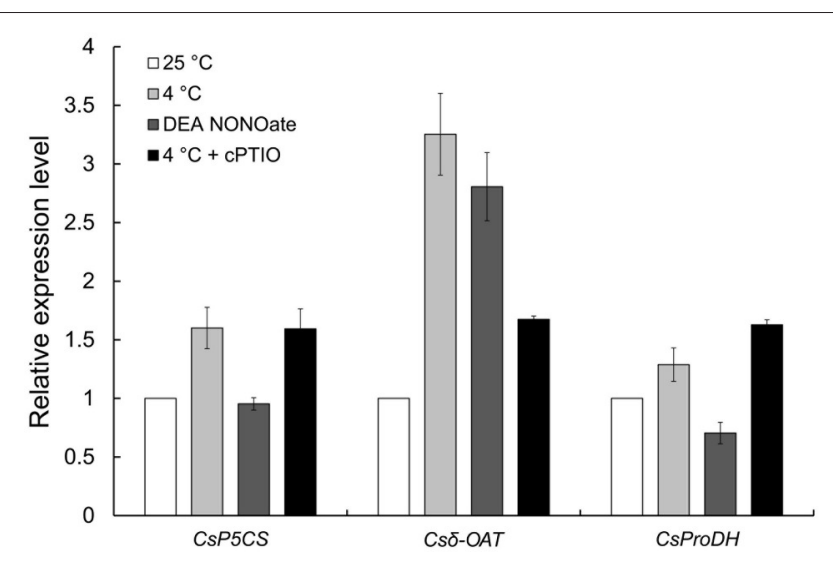

FIGURE 10 | The expression levels of three rate-limiting enzyme genes in Pro metabolism were analyzed by qRT-PCR. Cold-induced NO promotes the expression of Cs $\delta$-OAT and inhibits the expression of CsProDH but does not affect the expression of CSP5CS.

cold stress may be the result of the synergism of several pathways in C. sinensis pollen tubes.

As the most ubiquitous second messenger, $\mathrm{Ca}^{2+}$ dependent signaling networks can respond to many physiological processes in plant cells, and it has been shown that there is a close coupling between the intracellular tip-focused $\mathrm{Ca}^{2+}$ gradient and the polarized growth of the pollen tube (Holdaway-Clarke and Hepler, 2003). Currently, increasing evidence confirms that there is complicated crosstalk among $\mathrm{NO}$ and $\mathrm{Ca}^{2+}$ signaling pathways. Prado et al. (2004) reported that a putative NO-cGMP signaling pathway induced pollen tube reorientation through effects on cytoplasmic $\mathrm{Ca}^{2+}$ concentrations in lily. Similarly, $\mathrm{NO}$ was also found to modulate the cytoplasmic $\mathrm{Ca}^{2+}$ gradient to regulate Pinus bungeana pollen tube development largely by mediating $\mathrm{Ca}^{2+}$ influx, which is most likely dependent on cGMPactivated channels in pollen tubes (Wang et al., 2009). In the present study, we found that the $\mathrm{Ca}^{2+}$ gradient was disrupted by both cold stress and NO, and the disruption was relieved by cPTIO. Combined with our previous reports (Wang et al., 2012), it is reasonable to speculate that cold-induced NO inhibits the polarized growth of $C$. sinensis pollen tubes dependent on the damage to the cytoplasmic $\mathrm{Ca}^{2+}$ gradient at the pollen tube tip zone. Additionally, pharmacological and biochemical studies have shown that NO signaling in plants is mediated by cGMP (Prado et al., 2004) and cyclic nucleotide-gated ion channels (CNGCs), which are permeable to both monovalent and divalent cations (typically $\mathrm{K}^{+}, \mathrm{Na}^{+}$, and $\mathrm{Ca}^{2+}$ ) and are activated by cGMP and/or cAMP (Wang et al., 2013). Our data indicate that the expression of multiple CNGC genes is up-regulated after NO or cold stress treatment and that the expression of a large number of genes that encode proteins that activate CNGCs is induced, such as protein tyrosine kinases (PTKs) and protein tyrosine phosphatases (PTPs), which regulate the sensitivity of CNGCs on cGMP by catalytic phosphorylation (Chae et al., 2007). Recently, glutamate receptor-like channels (GLRs), a putative group of pollen $\mathrm{Ca}^{2+}$ channels, were identified, and their $\mathrm{Ca}^{2+}$ transport activities in pollen tubes have been confirmed based on direct electrophysiological, pharmacological and genetic evidence (Konrad et al., 2011; Michard et al., 2011). Interestingly, two GLR genes were identified from DEGs of CK-Vs-LT and CKVs-NO, implying that GRLs participate in the process in which $\mathrm{NO}$ results in a $\mathrm{Ca}^{2+}$ gradient disruption at the pollen tube tip after treatment with cold stress. Furthermore, the expression of another class of $\mathrm{Ca}^{2+}$ channel protein family genes, two pore calcium channel protein genes (TPC, Unigene1219_All), was shown to be up-regulated after NO or cold stress treatment, and the induction of cold stress was inhibited by cPTIO, indicating that TPC was also involved in the regulatory process of cold-induced $\mathrm{NO}$ disruption of the $\mathrm{Ca}^{2+}$ gradient at the pollen tube tip. These data suggest that NO regulates the cytoplasmic $\mathrm{Ca}^{2+}$ gradient largely by mediating $\mathrm{Ca}^{2+}$ fluxes under cold stress, which is most likely dependent on various $\mathrm{Ca}^{2+}$ channels in C. sinensis pollen tubes, such as CNGCs, GLRs, and TPCs.

The downstream proteins that can bind $\mathrm{Ca}^{2+}$ and act upon changes in $\mathrm{Ca}^{2+}$ concentrations to perform specific functions play important roles in the process of pollen tube tip growth (Konrad et al., 2011), particularly $\mathrm{Ca}^{2+}$ sensor and relay proteins, including calmodulin (CaM), CaM-like proteins (CMLs), $\mathrm{Ca}^{2+}$ dependent protein kinases (CDPKs), calcineurin B-like proteins (CBLs), and CBL-interacting protein kinases (CIPKs; Gutermuth et al., 2013; Steinhorst and Kudla, 2013; Zhou et al., 2014). Recently, Zhou et al. (2015) reported that Arabidopsis CIPK19 is required for pollen tube growth and polarity by participating in $\mathrm{Ca}^{2+}$ homeostasis dependent on the modulation of $\mathrm{Ca}^{2+}$ influx. In our investigation, we also found that multiple sensors and relay protein genes were up/down-regulated in C. sinensis pollen tubes in response to $\mathrm{NO}$ or cold stress treatment. Combined with previous results, we infer that $\mathrm{Ca}^{2+}$ binging proteins are involved in cold-induced NO-inhibited pollen tube tip growth, which is dependent on the regulation of NO on the cytoplasmic $\mathrm{Ca}^{2+}$ gradient (Domingos et al., 2015). In addition, accumulating evidence indicates that cytoskeleton elements (actin/myosin cables) control cytoplasmic streaming, the distribution of the endoplasmic reticulum (ER) and the transport of secretory vesicles and that actin polymerization itself also contributes to pollen tube growth (Chen et al., 2007). Concurrently, the dynamic state of the cytoskeleton elements is controlled via numerous regulatory factors, including several actin-binding proteins activated in response to $\mathrm{Ca}^{2+}$ (Cárdenas et al., 2008). Moreover, Wang et al. (2009) reported that Factin organization in the tip region of pollen tubes sensitive to $\mathrm{NO}$ is partly dependent on the $\mathrm{Ca}^{2+}$ gradient during $\mathrm{NO}$ signaling in $P$. bungeana pollen tubes. Our results show that cold-induced NO stimulation caused a sharp decline in the number of vesicles and ER distribution abnormality in the $C$. sinensis pollen tube tip region accompanied by a sharper $\mathrm{Ca}^{2+}$ gradient disruption. In addition, a large number of cytoskeletonrelated genes were up/down-regulated in this process, such as actin, formin-like protein genes, myosin-like genes, kinesin genes, actin-depolymerizing factor, and the caltractin gene, which encodes a type of $\mathrm{Ca}^{2+}$ binding protein. This suggests that vesicular trafficking and ER distribution were perturbed by the cold-induced NO accumulation, which may be partly dependent 
on the regulation of the NO-induced $\mathrm{Ca}^{2+}$ gradient change in cytoskeleton elements, particularly the dynamic polymerization status of F-actin (Chen et al., 2009). Furthermore, mitogenactivated protein kinases (MAPKs), which are another type of important downstream target of the $\mathrm{Ca}^{2+}$ signal, have been confirmed to mediate the guidance response in pollen tubes (Guan et al., 2014). In addition, accumulating evidence indicates that MAPKs function as intracellular targets for NO and participate in the developmental processes of plants, including pollen tube tip growth (Arasimowicz and Floryszak-Wieczorek, 2007; Domingos et al., 2015; Sanz et al., 2015). As expected, some MAPK genes and downstream genes were induced by colddependent NO in C. sinensis pollen tubes, which is consistent with a previous report showing that $\mathrm{NO}$ activates a potential MAPK during NO-induced PCD in A. thaliana suspension cultures (Clarke et al., 2000).

It is generally accepted that there is crosstalk among NO, $\mathrm{Ca}^{2+}$, and ROS signaling pathways in plants, particularly in the process of pollen tube growth and the response to environmental stress (Domingos et al., 2015). For example, NADPH oxidases (NOX) are expressed in pollen tubes and localize to the plasma membrane (PM) where they produce ROS, namely $\mathrm{H}_{2} \mathrm{O}_{2}$, which promotes NO synthesis through NR and/or NOA1. In contrast, NO activates protein kinases (PK), enabling NOX to bind $\mathrm{Ca}^{2+}$, triggering more ROS production, and NO-dependent cGMP activates $\mathrm{Ca}^{2+}$ channels at the PM to provide adequate $\mathrm{Ca}^{2+}$ for NOX (Liu et al., 2009; Wilkins et al., 2011; Wudick and Feijó, 2014; Domingos et al., 2015). In addition, the activation of PM NOX also can be triggered by the CDPK in a $\mathrm{Ca}^{2+}$ dependent manner to produce ROS in pollen tubes (Kobayashi et al., 2007; Potocký et al., 2007; Kaya et al., 2014). Our results show that NO increases the accumulation of ROS accompanied by a significantly up-regulated expression of the NOX gene and $\mathrm{Ca}^{2+}$ gradient disruption in the C. sinensis pollen tube in response to cold stress. Regardless of the reactivity of ROS and $\mathrm{NO}$, our results suggest that cold-induced NO regulates the production of ROS partly through the activation of PM NOX triggered by $\mathrm{Ca}^{2+}$, which further supports the results of previous studies (Kaya et al., 2014; Domingos et al., 2015). Furthermore, increasing evidence suggests that small GTPases in plants called RAC/ROPs (RACs are used in this study) function as molecular switches in the polarized cell growth of pollen tubes and root hairs and in defense-related responses (Zou et al., 2011; Huang et al., 2014). Kost et al. (1999) reported that the overexpression of RAC1 enlarged the apical regions of the PM containing RAC1, which is correlated with the severity of the depolarized growth of pollen tubes. In the present study, the up-regulation of $R A C 1$ and the down-regulation of RAC5 were detected after treatment with $\mathrm{NO}$ or cold stress, and the effects of cold stress were relieved by cPTIO, implying that RACs play important roles in coldinduced NO-inhibited tip growth in C. sinensis pollen tubes, which supports the above results of Kost et al. (1999). In addition, recent studies have shown that RACs affect the actin dynamics mediated by $\mathrm{Ca}^{2+}$ and/or ADF, thereby controlling the polarized growth of pollen tubes (Chen et al., 2003; Fu, 2010; Wu et al., 2011). Moreover, RACs are found to be involved in stimulating the ROS accumulation in the polarized growth of pollen tubes (Potocký et al., 2012; Kaya et al., 2014). Given the crosstalk among
$\mathrm{NO}, \mathrm{Ca}^{2+}$ and the ROS signaling pathway, we speculate that RACs are involved in the regulation of $\mathrm{NO}$ on $\mathrm{Ca}^{2+}$ and the ROS signaling pathway in the process of cold stress inhibiting the $C$. sinensis pollen tube tip growth.

Recently, Wilkins et al. (2015) reported that cytosolic pH $\left([\mathrm{pH}]_{c y t}\right)$ acidification was necessary and sufficient for triggering several key hallmark features of the self-incompatibility-induced PCD signaling pathway. Our data also reveal that coldinduced NO inhibits the tip growth of $C$. sinensis pollen tubes accompanied by a significant $[\mathrm{pH}]_{c y t}$ acidification, which confirms the role of $\mathrm{pH}$ signaling in plant pollen tube growth (Michard et al., 2009). In addition, previous studies have suggested that the changes in $[\mathrm{pH}]_{c y t}$ in the pollen tube tip are dependent on $\mathrm{H}^{+}$fluxes mediated by the regulation of $\mathrm{Ca}^{2+}$ on PM H $\mathrm{H}^{+}$-ATPase and $\mathrm{Na}^{+} / \mathrm{H}^{+}$antiporter (SOS1) activities (Certal et al., 2008; Michard et al., 2008; Guo et al., 2009). Interestingly, whereas cold-induced $\mathrm{NO}$ results in C. sinensis pollen tube tip acidification, the expression of $\mathrm{PM} \mathrm{H}^{+}$-ATPase and the $\mathrm{Na}^{+} / \mathrm{H}^{+}$antiporter gene are found to be significantly upregulated, suggesting that potential $\mathrm{H}^{+}$fluxes participate in the process of pollen tube tip acidification, which is similar to reports by Sun et al. (2009) that the synergistic effect between $\mathrm{PM} \mathrm{H}^{+}$ATPase and the $\mathrm{Na}^{+} / \mathrm{H}^{+}$antiporter increased $\mathrm{H}^{+}$influx in root protoplasts under salt stress. Of course, the contribution of $\mathrm{Ca}^{2+}$ cannot be neglected in the above process (Michard et al., 2009). Furthermore, $\mathrm{pH}$ as a regulator of PME activity was involved in the regulation of PME on pectin status and distribution at the pollen tube cell wall (Li et al., 1994). Increasing evidence confirms that the dynamic balance between cell wall extensibility and rigidity is another key factor that regulates tip growth in pollen tubes (Chen et al., 2007, 2009). In the present study, we also found that two PME genes were up-regulated after treatment with NO or cold stress. In addition, immunolabeling with LM19 and LM20 showed that cold-induced NO changed the distribution of acidic and esterified pectins at the cell wall of $C$. sinensis pollen tubes compared to that in the controls; this is similar to data showing that an increase in the degree of cell wall rigidity and a decrease in visco-elasticity influence pollen tube growth and architecture (Parre and Geitmann, 2005; Wang et al., 2009). The characteristic dot-strengthened and ring-like structures of AGPs at pollen tubes cell wall disappeared after treatment with NO or cold stress, which is similar to the results of Chen et al. (2007). In addition, ultrastructure observation showed that cold-induced NO causes cell wall thickening, smoothing and color deepening, which is likely dependent on the above changes in cell wall construction. Taking these findings together, we speculate that cold-induced NO stimulates changes in cell wall component distributions, leading to excess wall rigidity at the tip of the pollen tube, thereby inhibiting the polarized growth of the C. sinensis pollen tube tip, which may partly account for the synergistic effect of $\mathrm{pH}$ and PME mediated by NO.

Furthermore, it is well known that higher plants accumulate free Pro in response to a number of abiotic stresses, such as drought, salinity and freezing (Zhao et al., 2009). Nevertheless, only a few reports have indicated that NO and Pro cross-talk in cold acclimation and freezing tolerance. For example, Zhao et al. (2009) showed that cold-induced NO acts as a signal to evoke Pro accumulation via enhanced synthesis and reduced 
degradation by regulating related genes of the Pro biosynthetic pathway in Arabidopsis, which may be a function of $\mathrm{NO}$ in freezing tolerance. Similarly, our previous study showed that NO participated in stimulating Pro accumulation in C. sinensis pollen tube responses to cold stress (Wang et al., 2012). In addition, Ruan et al. (2004) reported that NO promoted the activity of P5CS1 and decreased the activity of ProDH. Interestingly, our present study showed that cold-induced NO increased the expression of $C s \delta$-OAT and reduced the expression of $\mathrm{CsProDH}$ but had no effect on the expression of CsP5CS (Figure 10). These results reveal that $\mathrm{NO}$ regulated Pro accumulation by increasing the expression of $C s \delta-O A T$ instead of CsP5CS and by reducing the expression of $\mathrm{Cs} P \mathrm{PoDH}$ in $\mathrm{C}$. sinensis pollen tubes during the response to cold stress, although the contribution of CsP5CS cannot be ignored in cold stress-induced Pro accumulation (Figure S6).

In summary, our cytological and transcriptomic analyses provide a more global picture of the role of $\mathrm{NO}$ in cold stress to inhibit the polarized tip growth of $C$. sinensis pollen tubes. A complex signaling network dominated by NO, including $\mathrm{Ca}^{2+}$, ROS, $\mathrm{pH}, \mathrm{RACs}$ signaling, and the crosstalk among them, was investigated in the $C$. sinensis pollen tube response to cold stress, which is summarized in Figure 11. This study provided two novel findings. First, cold-induced NO causes $\mathrm{Ca}^{2+}$ gradient disruption in C. sinensis pollen tubes, most likely through $\mathrm{Ca}^{2+}$ fluxes mediated by various $\mathrm{Ca}^{2+}$ channels and through subsequently triggered secondary and tertiary regulatory networks, including $\mathrm{Ca}^{2+}$ sensor and relay proteins, the MAPK cascade, ROS and $\mathrm{pH}$ signaling. Second, $[\mathrm{pH}]_{c y t}$ acidification interacted with PMEs, leading to changes in the cell wall structure and component distributions, thereby inhibiting the polarized growth of the $C$. sinensis pollen tube tips after treatment with cold stress. Furthermore, RAC signaling is involved in the process of cold-induced NO-inhibited C. sinensis pollen tube polarized growth, possibly through regulating the $\mathrm{Ca}^{2+}$ and ROS signaling

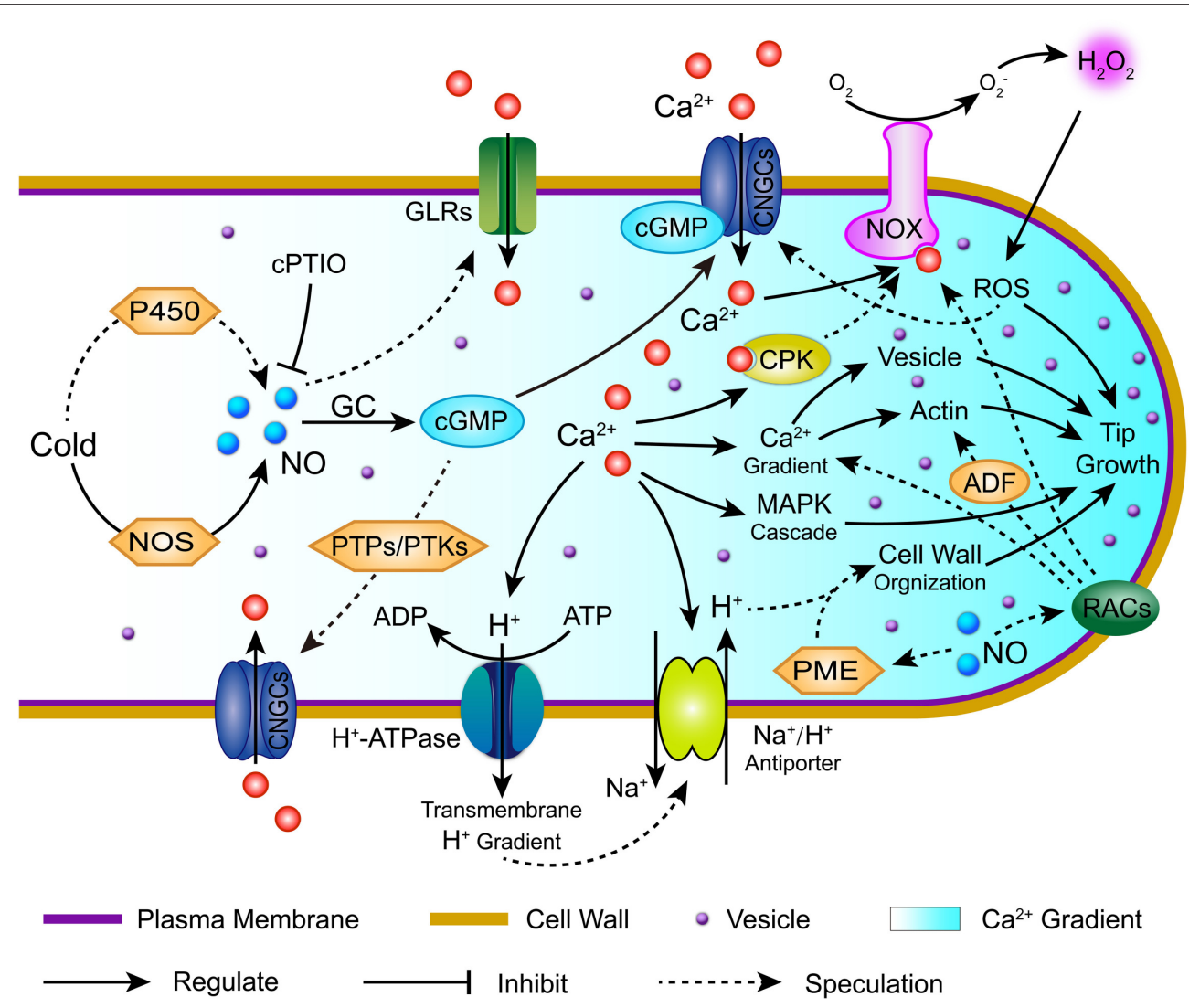

FIGURE 11 | Hypothetical model summarizing the potential signaling pathway of nitric oxide (NO) involved in cold-inhibited C. sinensis pollen tube growth. This simplified model was based on the pollen tube models proposed by Wang et al. (2009 and 2012), (Wudick and Feijó, 2014), and Domingos et al. (2015). Cold stress induces an increase in NO through the synergism of several pathways in C. sinensis pollen tubes, such as the accumulation of NOS-like activity and cytochrome P450 activity. Consequently, the cytoplasmic $\mathrm{Ca}^{2+}$ gradient was regulated largely by mediating the $\mathrm{Ca}^{2+}$ flux, which is dependent on various $\mathrm{Ca}^{2+}$ channels, such as CNGCs (cGMP-activated channels), GLRs and TPCs, and this subsequently triggered secondary and tertiary regulatory networks, including Ca ${ }^{2+}$ sensor and relay proteins, the MAPK cascade, ROS, actin, vesicles and pH signaling. In addition, $\mathrm{Ca}^{2+}$-dependent $[\mathrm{pH}]_{\text {cyt }}$ acidification interacted with PMEs, leading to changes in the cell wall structure and component distribution. Furthermore, RAC signaling involved in the process of cold-induced NO inhibited C. sinensis pollen tube polarized growth by regulating the $\mathrm{Ca}^{2+}$ and $\mathrm{ROS}$ signaling pathways. Together, the complex signaling network dominated by NO mediates the cold-inhibited $\mathrm{C}$. sinensis pollen tube growth. NOS, nitric oxide synthase; GC, guanylyl cyclase; PTPs, protein tyrosine phosphatases; cPTIO,

2-(4-carboxyphenyl)-4,4,5,5-tetramethylimidazoline-1-oxyl-3-oxide; PTKs, protein tyrosine kinases; CNGCs, cyclic nucleotide-gated ion channels; GLRs, glutamate receptor-like channels; CPK, calcium-dependent protein kinase; NOX, NADPH oxidase; ROS, reactive oxygen species; ADF, actin-depolymerizing factor; PME, pectin methylesterase; RACs, Rac-like GTP-binding proteins. 
pathways, which is also an interesting result. Taken together, our study provides new insights into the multifaceted mechanistic framework for the functions of NO in cold-inhibited C. sinensis pollen tube growth.

\section{AUTHOR CONTRIBUTIONS}

WW, YW, and XS designed research; WW, XS, ZS, DL, and YW performed research; WW, JP, XY, PC, and YW analyzed data; WW and YW wrote the paper; XL and YW revised this paper.

\section{REFERENCES}

Airaki, M., Leterrier, M., Mateos, R. M., Valderrama, R., Chaki, M., Barroso, J. B., et al. (2012). Metabolism of reactive oxygen species and reactive nitrogen species in pepper (Capsicum annuum L.) plants under low temperature stress. Plant Cell Environ. 35, 281-295. doi: 10.1111/j.1365-3040.2011.02310.x

Arasimowicz, M., and Floryszak-Wieczorek, J. (2007). Nitric oxide as a bioactive signalling molecule in plant stress responses. Plant Sci. 172, 876-887. doi: 10.1016/j.plantsci.2007.02.005

Cárdenas, L., Lovy-Wheeler, A., Kunkel, J. G., and Hepler, P. K. (2008). Pollen tube growth oscillations and intracellular calcium levels are reversibly modulated by actin polymerization. Plant Physiol. 146, 1611-1621. doi: 10.1104/pp.107.113035

Certal, A. C., Almeida, R. B., Carvalho, L. M., Wong, E., Moreno, N., Michard, E., et al. (2008). Exclusion of a proton ATPase from the apical membrane is associated with cell polarity and tip growth in Nicotiana tabacum pollen tubes. Plant Cell 20, 614-634. doi: 10.1105/tpc.106.047423

Chae, K. S., Ko, G. Y. P., and Dryer, S. E. (2007). Tyrosine phosphorylation of cGMP-gated ion channels is under circadian control in chick retina photoreceptors. Invest. Ophthalmol. Vis. Sci. 48, 901. doi: 10.1167/iovs.06-0824

Chen, C. Y. H., Cheung, A. Y., and Wu, H. M. (2003). Actin-depolymerizing factor mediates Rac/Rop GTPase-regulated pollen tube growth. Plant Cell 15, 237-249. doi: 10.1105/tpc.007153

Chen, T., Teng, N., Wu, X., Wang, Y., Tang, W., Šamaj, J., et al. (2007). Disruption of actin filaments by latrunculin B affects cell wall construction in Picea meyeri pollen tube by disturbing vesicle trafficking. Plant Cell Physiol. 48, 19-30. doi: $10.1093 / \mathrm{pcp} / \mathrm{pcl} 036$

Chen, T., Wu, X., Chen, Y., Li, X., Huang, M., Zheng, M., et al. (2009). Combined proteomic and cytological analysis of $\mathrm{Ca}^{2+}$-calmodulin regulation in Picea meyeri pollen tube growth. Plant Physiol. 149, 1111-1126. doi: 10.1104/pp.108.127514

Clarke, A., Desikan, R., Hurst, R. D., Hancock, J. T., and Neill, S. J. (2000). NO way back: nitric oxide and programmed cell death in Arabidopsis thaliana suspension cultures. Plant J. 24, 667-677. doi: 10.1046/j.1365313x.2000.00911.x

Domingos, P., Prado, A. M., Wong, A., Gehring, C., and Feijo, J. A. (2015). Nitric oxide: a multitasked signaling gas in plants. Mol. Plant 8, 506-520. doi: 10.1016/j.molp.2014.12.010

Fu, Y. (2010). The actin cytoskeleton and signaling network during pollen tube tip growth. J. Integr. Plant Biol. 52, 131-137. doi: 10.1111/j.1744-7909.2010.00922.x

Gao, Y. B., Wang, C. L., Wu, J. Y., Zhou, H. S., Jiang, X. T., Wu, J. Y., et al. (2014). Low temperature inhibits pollen tube growth by disruption of both tip-localized reactive oxygen species and endocytosis in Pyrus bretschneideri Rehd. Plant Physiol. Biochem. 74, 255-262. doi: 10.1016/j.plaphy.2013.11.018

Guan, Y., Lu, J., Xu, J., McClure, B., and Zhang, S. (2014). Two mitogen-activated protein kinases, MPK3 and MPK6, are required for funicular guidance of pollen tubes in Arabidopsis. Plant Physiol. 165, 528-533. doi: 10.1104/pp.113.231274

Guillas, I., Guellim, A., Rezé, N., and Baudouin, E. (2013). Long chain base changes triggered by a short exposure of Arabidopsis to low temperature are altered by AHb1 non-symbiotic haemoglobin overexpression. Plant Physiol. Biochem. 63, 191-195. doi: 10.1016/j.plaphy.2012.11.020

\section{ACKNOWLEDGMENTS}

This work was supported by the National Natural Science Foundation of China (grant No. 31370014, 31470690 and 31371387).

\section{SUPPLEMENTARY MATERIAL}

The Supplementary Material for this article can be found online at: http://journal.frontiersin.org/article/10.3389/fpls.2016. 00456

Guo, K. M., Babourina, O., and Rengel, Z. (2009). $\mathrm{Na}^{+} / \mathrm{H}^{+}$antiporter activity of the SOS1 gene: lifetime imaging analysis and electrophysiological studies on Arabidopsis seedlings. Physiol. Plant. 137, 155-165. doi: 10.1111/j.13993054.2009.01274.x

Gutermuth, T., Lassig, R., Portes, M. T., Maierhofer, T., Romeis, T., Borst, J. W., et al. (2013). Pollen tube growth regulation by free anions depends on the interaction between the anion channel SLAH3 and calciumdependent protein kinases CPK2 and CPK20. Plant Cell 25, 4525-4543. doi: $10.1105 /$ tpc.113.118463

Hedhly, A. (2011). Sensitivity of flowering plant gametophytes to temperature fluctuations. Environ. Exp. Bot. 74, 9-16. doi: 10.1016/j.envexpbot.2011.03.016

Holdaway-Clarke, T. L., and Hepler, P. K. (2003). Control of pollen tube growth: role of ion gradients and fluxes. New Phytol. 159, 539-563. doi: 10.1046/j.14698137.2003.00847.x

Huang, J. B., Liu, H., Chen, M., Li, X., Wang, M., Yang, Y., et al. (2014). ROP3 GTPase contributes to polar auxin transport and auxin responses and is important for embryogenesis and seedling growth in Arabidopsis. Plant Cell 26, 3501-3518. doi: 10.1105/tpc.114.127902

Kaya, H., Nakajima, R., Iwano, M., Kanaoka, M. M., Kimura, S., Takeda, S., et al. (2014). $\mathrm{Ca}^{2+}$-activated reactive oxygen species production by Arabidopsis RbohH and RbohJ is essential for proper pollen tube tip growth. Plant Cell 26, 1069-1080. doi: 10.1105/tpc.113.120642

Klemens, P. A., Patzke, K., Trentmann, O., Poschet, G., Büttner, M., Schulz, A., et al. (2014). Overexpression of a proton-coupled vacuolar glucose exporter impairs freezing tolerance and seed germination. New Phytol. 202, 188-197. doi: $10.1111 / \mathrm{nph} .12642$

Kobayashi, M., Ohura, I., Kawakita, K., Yokota, N., Fujiwara, M., Shimamoto, K., et al. (2007). Calcium-dependent protein kinases regulate the production of reactive oxygen species by potato NADPH oxidase. Plant Cell 19, 1065-1080. doi: $10.1105 /$ tpc. 106.048884

Konrad, K. R., Wudick, M. M., and Feijó, J. A. (2011). Calcium regulation of tip growth: new genes for old mechanisms. Curr. Opin. Plant Biol. 14, 721-730. doi: 10.1016/j.pbi.2011.09.005

Kost, B., Lemichez, E., Spielhofer, P., Hong, Y., Tolias, K., Carpenter, C., et al. (1999). Rac homologues and compartmentalized phosphatidylinositol 4, 5bisphosphate act in a common pathway to regulate polar pollen tube growth. $J$. Cell Biol. 145, 317-330. doi: 10.1083/jcb.145.2.317

Kováčik, J., Babula, P., Klejdus, B., Hedbavny, J., and Jarošova, M. (2014). Unexpected behavior of some nitric oxide modulators under cadmium excess in plant tissue. PLoS ONE 9:e91685. doi: 10.1371/journal.pone.0091685

Lanteri, M. L., Laxalt, A. M., and Lamattina, L. (2008). Nitric oxide triggers phosphatidic acid accumulation via phospholipase D during auxin-induced adventitious root formation in cucumber. Plant Physiol. 147, 188-198. doi: 10.1104/pp.107.111815

Li, Y., Chen, F., Linskens, H., and Cresti, M. (1994). Distribution of unesterified and esterified pectins in cell walls of pollen tubes of flowering plants. Sex. Plant Reprod. 7, 145-152. doi: 10.1007/BF00228487

Liao, W. B., Huang, G. B., Yu, J. H., and Zhang, M. L. (2012). Nitric oxide and hydrogen peroxide alleviate drought stress in marigold explants and promote its adventitious root development. Plant Physiol. Biochem. 58, 6-15. doi: 10.1016/j.plaphy.2012.06.012 
Liu, P., Li, R. L., Zhang, L., Wang, Q. L., Niehaus, K., Baluška, F., et al. (2009). Lipid microdomain polarization is required for NADPH oxidase-dependent ROS signaling in Picea meyeri pollen tube tip growth. Plant J. 60, 303-313. doi: 10.1111/j.1365-313X.2009.03955.x

Liu, Y., Jiang, H., Zhao, Z., and An, L. (2010). Nitric oxide synthase like activitydependent nitric oxide production protects against chilling-induced oxidative damage in Chorispora bungeana suspension cultured cells. Plant Physiol. Biochem. 48, 936-944. doi: 10.1016/j.plaphy.2010.09.001

Livak, K. J., and Schmittgen, T. D. (2001). Analysis of relative gene expression data using real-time quantitative PCR and the $2^{-\Delta \Delta C T}$ method. Methods 25, 402-408. doi: 10.1006/meth.2001.1262

Majláth, I., Szalai, G., Soós, V., Sebestyén, E., Balázs, E., Vanková, R., et al. (2012). Effect of light on the gene expression and hormonal status of winter and spring wheat plants during cold hardening. Physiol. Plant. 145, 296-314. doi: 10.1111/j.1399-3054.2012.01579.x

Maruyama, K., Urano, K., Yoshiwara, K., Morishita, Y., Sakurai, N., Suzuki, H., et al. (2014). Integrated analysis of the effects of cold and dehydration on rice metabolites, phytohormones, and gene transcripts. Plant Physiol. 164, 1759-1771. doi: 10.1104/pp.113.231720

Michard, E., Alves, F., and Feijó, J. A. (2009). The role of ion fluxes in polarized cell growth and morphogenesis: the pollen tube as an experimental paradigm. Int. J. Dev. Biol. 53, 1609. doi: 10.1387/ijdb.072296em

Michard, E., Dias, P., and Feijó, J. A. (2008). Tobacco pollen tubes as cellular models for ion dynamics: improved spatial and temporal resolution of extracellular flux and free cytosolic concentration of calcium and protons using pHluorin and YC3. 1 CaMeleon. Sex. Plant Reprod. 21, 169-181. doi: 10.1007/s00497-008-0076-X

Michard, E., Lima, P. T., Borges, F., Silva, A. C., Portes, M. T., Carvalho, J. E., et al. (2011). Glutamate receptor-like genes form $\mathrm{Ca}^{2+}$ channels in pollen tubes and are regulated by pistil D-serine. Science 332, 434-437. doi: $10.1126 /$ science. 1201101

Neill, S., Barros, R., Bright, J., Desikan, R., Hancock, J., Harrison, J., et al. (2008). Nitric oxide, stomatal closure, and abiotic stress. J. Exp. Bot. 59, 165-176. doi: $10.1093 /$ jxb/erm 293

Parre, E., and Geitmann, A. (2005). Pectin and the role of the physical properties of the cell wall in pollen tube growth of Solanum chacoense. Planta 220, 582-592. doi: 10.1007/s00425-004-1368-5

Potocký, M., Jones, M. A., Bezvoda, R., Smirnoff, N., and žárský, V. (2007). Reactive oxygen species produced by NADPH oxidase are involved in pollen tube growth. New Phytol. 174, 742-751. doi: 10.1111/j.1469-8137.2007. 02042.x

Potocký, M., Pejchar, P., Gutkowska, M., Jiménez-Quesada, M. J., Potocká, A., de Dios Alché, J., et al. (2012). NADPH oxidase activity in pollen tubes is affected by calcium ions, signaling phospholipids and Rac/Rop GTPases. J. Plant Physiol. 169, 1654-1663. doi: 10.1016/j.jplph.2012.05.014

Prado, A. M., Colaço, R., Moreno, N., Silva, A. C., and Feij,ó, J. A. (2008). Targeting of pollen tubes to ovules is dependent on nitric oxide (NO) signaling. Mol. Plant 1, 703-714. doi: 10.1093/mp/ssn034

Prado, A. M., Porterfield, D. M., and Feijó, J. A. (2004). Nitric oxide is involved in growth regulation and re-orientation of pollen tubes. Development 131, 2707-2714. doi: 10.1242/dev.01153

Puyaubert, J., and Baudouin, E. (2014). New clues for a cold case: nitric oxide response to low temperature. Plant Cell Environ. 37, 2623-2630. doi: $10.1111 /$ pce. 12329

Qiao, W., and Fan, L. M. (2008). Nitric oxide signaling in plant responses to abiotic stresses. J. Integr. Plant Biol. 50, 1238-1246. doi: 10.1111/j.17447909.2008.00759.x

Qiao, W., Li, C., and Fan, L. M. (2014). Cross-talk between nitric oxide and hydrogen peroxide in plant responses to abiotic stresses. Environ. Exp. Bot. 100, 84-93. doi: 10.1016/j.envexpbot.2013.12.014

Qiao, W., Xiao, S., Yu, L., and Fan, L. M. (2009). Expression of a rice gene OsNOA1 re-establishes nitric oxide synthesis and stress-related gene expression for salt tolerance in Arabidopsis nitric oxide-associated 1 mutant Atnoa1. Environ. Exp. Bot. 65, 90-98. doi: 10.1016/j.envexpbot.2008.06.002

Ren, L., Sun, J., Chen, S., Gao, J., Dong, B., Liu, Y., et al. (2014). A transcriptomic analysis of Chrysanthemum nankingense provides insights into the basis of low temperature tolerance. BMC Genomics 15:844. doi: 10.1186/1471-2164$15-844$
Ruan, H. H., Shen, W. B., and Xu, L. L. (2004). Nitric oxide involved in the abscisic acid induced proline accumulation in wheat seedling leaves under salt stress. Acta Bot. Sin. 46, 1307-1315. Available online at: http://www.jipb.net/pubsoft/ content/2/3528/x040080.pdf

Sanz, L., Albertos, P., Mateos, I., Sánchez-Vicente, I., Lechón, T., FernándezMarcos, M., et al. (2015). Nitric oxide (NO) and phytohormones crosstalk during early plant development. J. Exp Bot. 66, 2857-2868. doi: $10.1093 /$ jxb/erv213

Saxena, I., and Shekhawat, G. (2013). Nitric oxide (NO) in alleviation of heavy metal induced phytotoxicity and its role in protein nitration. Nitric Oxide 32, 13-20. doi: 10.1016/j.niox.2013.03.004

Sehrawat, A., Abat, J. K., and Deswal, R. (2013a). RuBisCO depletion improved proteome coverage of cold responsive S-nitrosylated targets in Brassica juncea. Front Plant Sci. 4:342. doi: 10.3389/fpls.2013.00342

Sehrawat, A., Gupta, R., and Deswal, R. (2013b). Nitric oxide-cold stress signalling cross-talk, evolution of a novel regulatory mechanism. Proteomics 13, 1816-1835. doi: 10.1002/pmic.201200445

Sheng, X., Hu, Z., Lü, H., Wang, X., and Baluška, F., Šamaj, J. et al. (2006). Roles of the ubiquitin/proteasome pathway in pollen tube growth with emphasis on MG132-induced alterations in ultrastructure, cytoskeleton, and cell wall components. Plant Physiol. 141, 1578-1590. doi: 10.1104/pp.106. 081703

Spinelli, K. J., and Gillespie, P. G. (2012). Monitoring intracellular calcium ion dynamics in hair cell populations with Fluo-4 AM. PLoS ONE 7:e51874. doi: 10.1371/journal.pone.0051874

Srinivasan, A., Saxena, N., and Johansen, C. (1999). Cold tolerance during early reproductive growth of chickpea (Cicer arietinum L.): genetic variation in gamete development and function. Field Crop. Res. 60, 209-222. doi: 10.1016/S0378-4290(98)00126-9

Steinhorst, L., and Kudla, J. (2013). Calcium and reactive oxygen species rule the waves of signaling. Plant Physiol. 163, 471-485. doi: 10.1104/pp.113.222950

Sun, J., Chen, S., Dai, S., Wang, R., Li, N., Shen, X., et al. (2009). NaClinduced alternations of cellular and tissue ion fluxes in roots of saltresistant and salt-sensitive poplar species. Plant Physiol. 149, 1141-1153. doi: 10.1104/pp.108.129494

Tossi, V., Lamattina, L., and Cassia, R. (2009). An increase in the concentration of abscisic acid is critical for nitric oxide-mediated plant adaptive responses to UV-B irradiation. New Phytol. 181, 871-879. doi: 10.1111/j.14698137.2008.02722.x

Wang, W. D., Wang, Y. H., Du, Y. L., Zhao, Z., Zhu, X. J., Jiang, X., et al. (2014). Overexpression of Camellia sinensis H1 histone gene confers abiotic stress tolerance in transgenic tobacco. Plant Cell Rep. 33, 1829-1841. doi: 10.1007/s00299-014-1660-1

Wang, Y. F., Munemasa, S., Nishimura, N., Ren, H. M., Robert, N., Han, M., et al. (2013). Identification of cyclic GMP-activated nonselective $\mathrm{Ca}^{2+}$-permeable cation channels and associated CNGC5 and CNGC6 genes in Arabidopsis guard cells. Plant Physiol. 163, 578-590. doi: 10.1104/pp.113.225045

Wang, Y. H., Li, X. C., Zhu-Ge, Q., Jiang, X., Wang, W. D., Fang, W. P., et al. (2012). Nitric oxide participates in cold-inhibited Camellia sinensis pollen germination and tube growth partly via cGMP in vitro. PLoS ONE 7:e52436. doi: 10.1371/journal.pone.0052436

Wang, Y., Chen, T., Zhang, C., Hao, H., Liu, P., Zheng, M., et al. (2009) Nitric oxide modulates the influx of extracellular $\mathrm{Ca}^{2+}$ and actin filament organization during cell wall construction in Pinus bungeana pollen tubes. New Phytol. 182, 851-862. doi: 10.1111/j.1469-8137.2009.02820.x

Wilkins, K. A., Bancroft, J., Bosch, M., Ings, J., Smirnoff, N., and Franklin-Tong, V. E. (2011). Reactive oxygen species and nitric oxide mediate actin reorganization and programmed cell death in the self-incompatibility response of papaver. Plant Physiol. 156, 404-416. doi: 10.1104/pp.110.167510

Wilkins, K. A., Bosch, M., Haque, T., Teng, N., Poulter, N. S., and FranklinTong, V. E. (2015). Self-incompatibility-induced programmed cell death in Field poppy pollen involves dramatic acidification of the incompatible pollen tube cytosol. Plant Physiol. 167, 766-779. doi: 10.1104/pp.114.252742

Wilkins, K. A., Poulter, N. S., and Franklin-Tong, V. E. (2014). Taking one for the team: self-recognition and cell suicide in pollen. J. Exp. Bot. 65, 1331-1342. doi: $10.1093 /$ jxb/ert468

Wu, H., Hazak, O., Cheung, A. Y., and Yalovsky, S. (2011). RAC/ROP GTPases and auxin signaling. Plant Cell 23, 1208-1218. doi: 10.1105/tpc.111.083907 
Wu, J. Y., Jin, C., Qu, H. Y., Tao, S. T., Xu, G. H., Wu, J., et al. (2012). Low temperature inhibits pollen viability by alteration of actin cytoskeleton and regulation of pollen plasma membrane ion channels in Pyrus pyrifolia. Environ. Exp. Bot. 78, 70-75. doi: 10.1016/j.envexpbot.2011.12.021

Wudick, M. M., and Feijó, J. A. (2014). At the intersection: merging $\mathrm{Ca}^{2+}$ and ROS signaling pathways in pollen. Mol. Plant 7, 1595-1597. doi: 10.1093/mp/ ssu096

Xuan, Y., Zhou, S., Wang, L., Cheng, Y., and Zhao, L. (2010). Nitric oxide functions as a signal and acts upstream of AtCaM3 in thermotolerance in Arabidopsis seedlings. Plant Physiol. 153, 1895-1906. doi: 10.1104/pp.110.160424

Zhao, M. G., Chen, L., Zhang, L. L., and Zhang, W. H. (2009). Nitric reductase-dependent nitric oxide production is involved in cold acclimation and freezing tolerance in Arabidopsis. Plant Physiol. 151, 755-767. doi: 10.1104/pp.109.140996

Zhao, M. G., Tian, Q. Y., and Zhang, W. H. (2007). Nitric oxide synthasedependent nitric oxide production is associated with salt tolerance in Arabidopsis. Plant Physiol. 144, 206-217. doi: 10.1104/pp.107. 096842

Zhao, Y., Li, J., Liu, H., Xi, Y., Xue, M., Liu, W., et al. (2015). Dynamic transcriptome profiles of skeletal muscle tissue across 11 developmental stages for both Tongcheng and Yorkshire pigs. BMC Genomics 16:377. doi: 10.1186/s12864-015-1580-7
Zhou, L., Lan, W., Chen, B., Fang, W., and Luan, S. (2015). A Calcium SensorRegulated Protein Kinase CIPK19 is required for pollen tube growth and polarity1. Plant Physiol. 114:256065. doi: 10.1104/pp.114.256065

Zhou, L., Lan, W., Jiang, Y., Fang, W., and Luan, S. (2014). A calcium-dependent protein kinase interacts with and activates a calcium channel to regulate pollen tube growth. Mol. Plant 7, 369-376. doi: 10.1093/mp/sst125

Zou, Y., Aggarwal, M., Zheng, W. G., Wu, H. M., and Cheung, A. Y. (2011) Receptor-like kinases as surface regulators for RAC/ROP-mediated pollen tube growth and interaction with the pistil. AoB Plants 2011:plr017. doi: 10.1093/aobpla/plr017

Conflict of Interest Statement: The authors declare that the research was conducted in the absence of any commercial or financial relationships that could be construed as a potential conflict of interest.

Copyright (c) 2016 Wang, Sheng, Shu, Li, Pan, Ye, Chang, Li and Wang. This is an open-access article distributed under the terms of the Creative Commons Attribution License (CC BY). The use, distribution or reproduction in other forums is permitted, provided the original author(s) or licensor are credited and that the original publication in this journal is cited, in accordance with accepted academic practice. No use, distribution or reproduction is permitted which does not comply with these terms. 